\title{
X-RAY PRODUCTION BY ION BOMBARDMENT
}

\author{
W. HINK \\ Physikalisches Institut der Universität Würzburg, D-87 Würzburg, R. F. A.
}

\begin{abstract}
Résumé. - On donne une brève revue des résultats expérimentaux et théoriques sur les rayons $\mathrm{X}$ produits par bombardement ionique. On peut conclure des données expérimentales que le mécanisme d'excitation des couches internes des atomes est différent pour des collisions d'ions lourds et de protons. Ces dernières sont décrites de façon satisfaisante dans l'approximation de Born avec des ondes planes et dans l'approximation classique des rencontres binaires. L'accord avec l'expérience est satisfaisant sauf en-dessous de $100 \mathrm{keV}$.

Pendant les collisions entre ions lourds et atomes, on suppose que l'interprétation des couches internes de l'ion et de l'atome produit une quasi-molécule et que des électrons des couches internes sont amenés dans les couches supérieures en laissant des trous dans les couches internes après la séparation du projectile et de l'atome cible. Ces trous peuvent être remplis avec émission de rayons $\mathrm{X}$ ou d'électrons Auger.

On compare, à la lumière des résultats récents, le cas des ions lourds à celui des protons. On peut noter des sections efficaces beaucoup plus grandes pour des vitesses comparables des projectiles, des décalages en énergie et des élargissement de raies dans le spectre de rayons $\mathrm{X}$, des lois de variation du rendement de fluorescence avec l'énergie de l'ion différentes.

Pour l'excitation de rayons $\mathrm{X}$, les ions lourds offrent dans certains cas des avantages considérables sur les photons ou les électrons : rendement élevé, sélectivité et toujours rapports pic sur bruit de fond élevés. On donne avec quelques détails, des exemples d'utilisation des rayons X produits par des ions lourds en l'analyse, traçage d'éléments et détection d'ions implantés.
\end{abstract}

\begin{abstract}
The available experimental and theoretical results on ion induced X-rays are briefly surveyed. From experimental data it may be concluded that the mechanism for inner shell excitation by heavy ion-atom collisions is different from that for proton-atom collisions. The latter are described successfully in Plane Wave Born Approximation (PWBA) and in classical Binary Encounter Approximation (BEA), leading to a general agreement with experiment except in the region below about $100 \mathrm{keV}$, where experimental data begin to fall short of the theoretical approaches. In the case of heavy ion-atom collisions it is supposed that during the collisions resulting from the interpenetration of the atomic shells of the colliding particles a quasimolecule is formed and inner shell electrons are promoted to higher shells leaving inner shell vacancies after the separation of projectile and target atom, which are filled under emission of $\mathrm{X}$-rays or Auger-electrons alternatively.

The important distinction of heavy ion-atom as compared to proton-atom collisions such as much higher cross-sections for comparable velocities of the projectiles, apparent energy shift and line broadening in the X-ray spectra, dependence of fluorescence yield on the energy of the impinging ion are discussed in connection with recent results.

As ion-induced X-rays offer in certain cases some extreme advantages over electron and photon methods namely high yield, selectivity and in any case high peak-to-background ratios, examples of the use of ion induced X-rays as an analytical tool are given such as elemental tracing and implanted ion detection.
\end{abstract}

1. Introduction. - It has been known since 1912 that characteristic X-rays are excited by the impact of high velocity ions on a target [1]. But little use has been made of the phenomenon. Certainly this is due to the fact that the X-ray yield (number of $\mathrm{K}, \mathrm{L}, \ldots \mathrm{X}$-ray photons emitted respectively from a target per impinging projectile and per unit solid angle) for ions is much smaller than for electrons of the same energy. In the last decade a strong interest in the generation of characteristic X-rays by ion impact has grown mainly for two reasons.

The first one is the suggestion by Sterk [2] to use ion produced X-rays for spectrochemical analysis. The inherent possibility for such an application of ioninduced X-rays may be demonstrated (Fig. 1) by an X-ray spectrum produced by $2.5 \mathrm{MeV}$ proton bombardment of air-borne particles spread out on pure carbon [3]. Although the use of rather large accelerators becomes necessary - the kinetic energy of protons, for example, must be roughly two orders of magnitude larger than that of electrons to achieve the same X-ray yield - there are some advantages of ion produced X-rays, such as the absence of continuous $\mathrm{X}$-rays (to be sure this holds only at impact energies below some hundred $\mathrm{keV}$ ) leading to a remarkable improvement of peak-to-background ratio of as much 


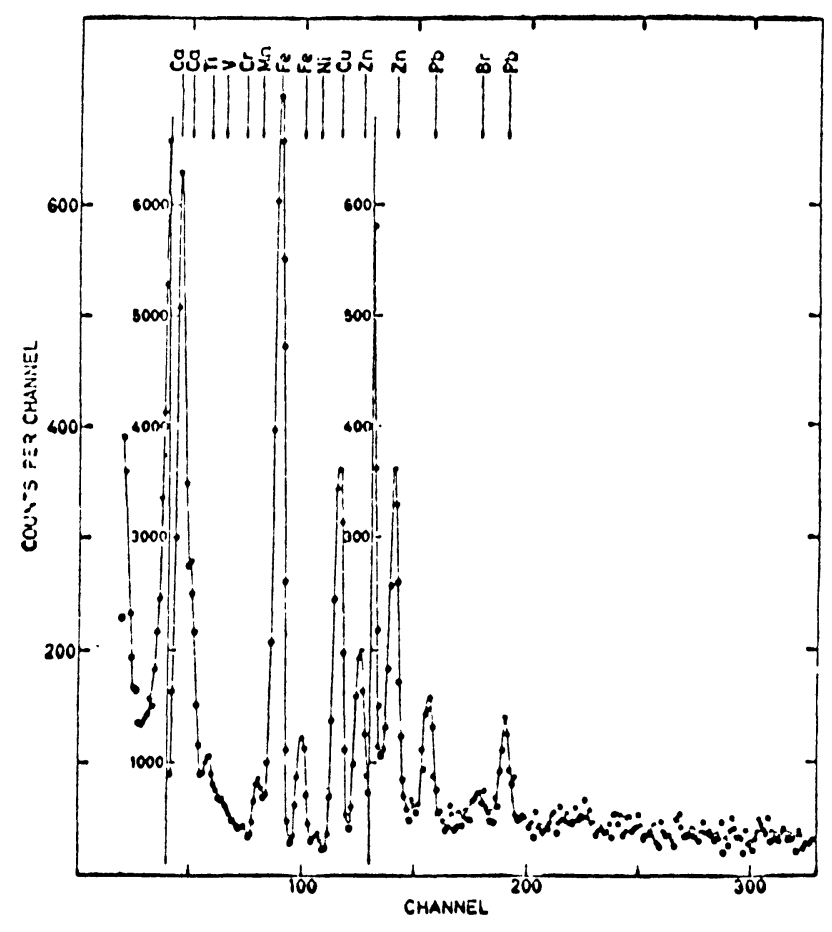

FIG. 1. - Pulse height spectrum taken with $[\mathrm{Si}(\mathrm{Li})]$-detector from X-ray analysis of air-borne particles spread on a pure carbon foil. The figure gives an example of the kind of information one gets in using proton-induced X-rays for analysis. The spectrum was obtained in $45 \mathrm{~min}$ and $1 \mathrm{mC}$ of $2.5 \mathrm{MeV}$-protons [3].

as two orders of magnitude [4] and the small effective sample size of the microprobe, which is ultimately limited to the range of the ions in the target [5]. Since ions of an appropriate energy have a range of only a few hundred Angströms in most solids, the probe may be used to examine surface deposits or to measure depth distributions of impurities.

The second reason for the increased interest in ionproduced X-rays lies in the progress made in the theoretical description of the fundamental inelastic collision process which may result in the inner-shell excitation of ionization followed alternatively by the emission of $\mathrm{X}$-ray photons or Auger-electrons. From the experimental X-ray yield-versus-energy curve of a thick solid target under ion bombardment, the inner-shell ionization cross-section may be extracted and compared with calculated cross-sections in different theoretical approaches.

In the MeV-region thin solid state target conditions allow an immediate extraction of the cross-sections, thus eliminating a large source of uncertainties in the extraction procedure. Gaseous target measurements which promise most reliability are still rare [6-10].

In a still growing number of studies the production of characteristic X-rays has been extended to a variety of heavy ion projectiles with energies ranging from the $\mathrm{keV}$-region up to high in the $\mathrm{MeV}$-region leading to rather unexpected results. These studies together with investigations of ion induced Auger-electron emission undertaken intensively since 1970 , provided new insights to the inelastic collision process. The crosssections for the production of X-rays by heavy ion impact are often higher by several orders of magnitude than those for the production by protons of the same relative velocity. These high yields cannot be explained by the direct ionization mechanism, which is relevant if the atomic number of the projectile $Z_{1}$ is much smaller than the atomic number $Z_{2}$ of the target atoms as in the case of proton impact. Another mechanism of ionization as proposed by U. Fano and W. Lichten [11] for the case of $\mathrm{Ar}^{+}$-Ar collisions is assumed if $Z_{1} \approx Z_{2}$. Inner atomic shell vacancies are produced by level crossing in the quasimolecule formed by the colliding particles. This phenomenon is of particular interest for spectrochemical analysis, because highly selective excitation of inner shells may occur by appropriate combination of projectile and target and choice of impact energy.

In this paper, a survey will be given of the field of ion produced X-rays relating to the production as to applications in spectrochemical analysis. As to the details of different processes and mechanisms leading to the production of characteristic X-rays by ion impact reference should be made to the Proceedings of the Int. Conf. on Inner Shell Ionization Phenomena [12] and to the comprehensive review paper on Inner Shell Vacancy Production in Ion-Atom collisions, published 1973 by J. D. Garcia, R. J. Fortner and T. M. Kavanagh in Review of Modern Physics [13]. These publications cover theoretical and experimental work relevant to this subject. Only the major features of this work will be presented here, rather special attention will be given to aspects which lend themselves to possible applications. The publications on applications are widely scattered in literature. Some of the recent studies showing the promising perspectives for future powerful applications of ion-induced X-rays will be discussed.

2. General. - At collision energies exceeding about $1 \mathrm{keV} /$ atomic mass unit (amu) the interaction between ion projectile and atoms is dominated by inelastic collisions which, especially in low collisions, lead to excitation and ionization of inner shells with a surprisingly high probability. If such an impact has removed an electron from an inner shell, the subsequent filling of the vacancy will lead to the emission of a characteristic X-ray photon or alternatively to the emission of an Auger-electron.

In addition to this process there are some more well understood primary processes that will lead to the emission of X-rays by ion impact : The emission of continuous X-rays (bremsstrahlung) due to the deflection of the impinging ions and secondary electrons in the Coulomb field of the target nuclei, the nuclear $\gamma$-ray emission and the emission of characteristic $\mathrm{X}$-rays by internal conversion, whenever the collidiny nuclei are Coulomb excited in the collision process. 
The socalled continuous molecular X-rays produced in two-fold collisions $[9,14,15]$ will be discussed in some detail in this paper.

All measurements on proton produced continuous $\mathrm{X}$-rays agree well with the quantummechanical predictions given by Sommerfeld and his colleagues (see ref. [1]). Thus the possible influence may be computed for every experimental arrangement. However, since the cross-section for the production of continuous $\mathrm{X}$-rays by protons as compared with electrons is reduced by the mass factor $(m / M)^{2}$ and depends on $Z_{2}^{2}$ $\left(Z_{2}\right.$ : atomic number of target atoms) the continuous $\mathrm{X}$-ray production is entirely negligible except for high $Z_{2}$ targets and proton energies in the MeV-region. For a discussion of ion induced continuous $\mathrm{X}$-rays see reference [15]. At impact energies below $\approx 1 \mathrm{MeV}$ the processes leading to continuous $\mathrm{X}$-rays can usually be neglected, but in analytical applications they can limit the ultimate sensitivity [16, 17, 75, 83].

If projectiles of kinetic energy $E$ are impinging normally on an infinitely thin target of thickness $\mathrm{d} x$, $N$ being the number of target atoms per unit volume, the number $\mathrm{d} Y_{\mathrm{K}, \mathrm{L}}, \ldots(E)$ of $\mathrm{K}, \mathrm{L}$, or other characte-

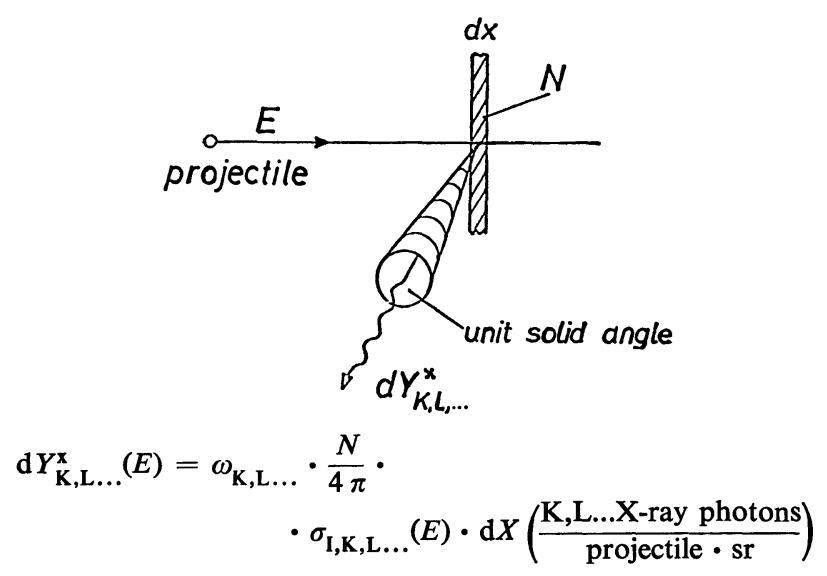

FIG. 2. - Scheme of thin target measurements.

ristic X-ray photons emitted respectively per ion per unit solid angle will be (Fig. 2)

$$
\begin{gathered}
\mathrm{d} Y_{\mathrm{K}, \mathrm{L}, \ldots}(E)=\omega_{\mathrm{K}, \mathrm{L}, \ldots} \cdot \frac{N}{4 \pi} \cdot \sigma_{\mathrm{I} ; \mathrm{K}, \mathrm{L}, \ldots}(E) \cdot \mathrm{d} x \\
\left(\frac{\mathrm{K}, \mathrm{L}, \ldots \text { X-ray photons }}{\text { projectile.sr }}\right)
\end{gathered}
$$

with $\sigma_{\mathrm{I} ; \mathrm{K}, \mathrm{L}, \ldots}(E)$ : cross section for the ionization in the $\mathrm{K}, \mathrm{L}, \ldots$ shell, respectively. The angular distribution of the characteristic X-radiation may be assumed to be isotropic $[1,18]$.

$$
\sigma_{\mathrm{X} ; \mathrm{K}, \mathrm{L}, \ldots}=\omega_{\mathrm{K}, \mathrm{L}, \ldots} \cdot \sigma_{\mathrm{I} ; \mathrm{K}, \mathrm{L}, \ldots}
$$

is sometimes called the $\mathrm{X}$-ray production cross-section. Measured $\sigma_{\mathbf{X}}$ values have an accuracy of typically a few percent. Because of the uncertainties in the fluorescence yield the accuracy of $\sigma_{\mathrm{I}}$ cannot be stated generally.
From eq. (1), which holds in the limit of very small energy loss of the ion in the target, one gets by integration over the whole path of the ion (taking into account the absorption of the X-rays within the target) the thick target yield $Y_{\mathrm{K}, \mathrm{L}}, \ldots\left(E_{0}\right)$. Here, in good approximation, a straight path of the projectile, stopped

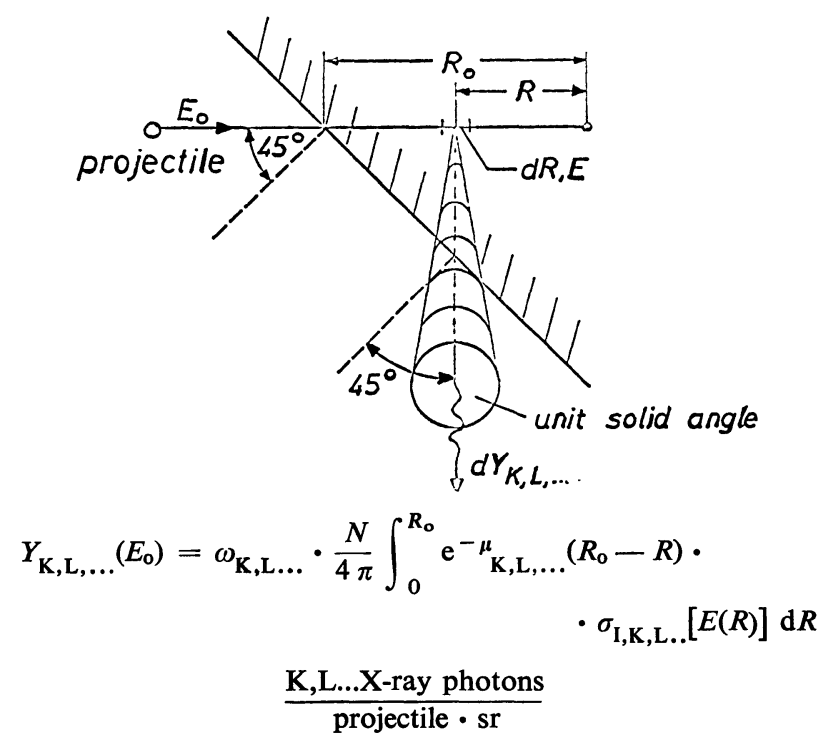

FIG. 3. - Scheme of thick target measurements.

within the target, is assumed. For the generally used geometry (Fig. 3) the X-ray yield results in

$$
\begin{aligned}
& Y_{\mathrm{K}, \mathrm{L}, \ldots}\left(E_{0}\right)=\omega_{\mathrm{K}, \mathrm{L}, \ldots} \cdot \frac{N}{4 \pi} \times \\
& \times \int_{0}^{R_{0}} \exp \left[-\mu_{\mathrm{K}, \mathrm{L}, \ldots}\left(R_{0}-R\right)\right] \cdot \sigma_{\mathrm{I} ; \mathrm{K}, \mathrm{L}, \ldots}[E(R)] \mathrm{d} R \\
& \left(\frac{\mathrm{K}, \mathrm{L}, \ldots \mathrm{X} \text {-ray photons }}{\text { projectile.sr }}\right)
\end{aligned}
$$

with $R_{0}$ : total range of an ion that impinges with kinetic energy $E_{0}$ on the target,

$R$ : residual range of the ion which has been slowed down in the target to a kinetic energy $E$, given by the energy-residual range relation $E(R)$,

$\mu_{\mathrm{K}, \mathrm{L}, \ldots}:$ linear attenuation coefficient of the target for the K, L, ... X-ray photons, respectively.

This yield as given by eq. (3) has been measured by most of the authors with typically the same accuracy as in thin target measurements.

By differentiation of the experimental thick target yield-versus-energy curve $Y_{\mathrm{K}, \mathrm{L}, \ldots .}\left(E_{0}\right)$, one extracts the ionization cross-section in a standard manner leading to formula

$$
\begin{aligned}
\sigma_{\mathrm{I} ; \mathrm{K}, \mathrm{L}, \ldots}\left(E_{0}\right)=\frac{4 \pi}{\omega_{\mathrm{K}, \mathrm{L}, \ldots} . N} \cdot & {\left[\left(\frac{\mathrm{d} Y_{\mathrm{K}, \mathrm{L}, \ldots . .}(E)}{\mathrm{d} E}\right)_{E_{0}} \cdot S\left(E_{0}\right)+\right.} \\
& \left.+\mu_{\mathrm{K}, \mathrm{L}, \ldots} \cdot Y_{\mathrm{K}, \mathrm{L}, \ldots}\left(E_{0}\right)\right]
\end{aligned}
$$


with

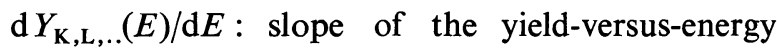
curve, and

$S\left(E_{0}\right)=(\mathrm{d} E / \mathrm{d} R)_{R_{0}}$ : stopping power of the target material for protons of energy $E_{0}$. Due to the uncertainties in stopping power in solid-absorption the extracted X-ray cross-sections have only an accuracy of typically $\pm 20-30 \%$.

3. Experimental. - The main components of the experimental equipment for measuring the yield of ionexcited X-rays are the following : ion source, accelerating systems, magnetic analyzer and beam collimator, which produce a well-collimated beam of ions that bombards the target atoms in a target chamber which acts simultaneously as a secondary electron shield by surrounding the target volume. Solid state targets are usually heatable thereby minimizing target surface contamination. In thick target measurements the target is preferably inclined $45^{\circ}$ with respect to the ion beam and the observation direction of the X-rays (Fig. 3). The number of incident ions is usually measured by a beam current integrator while simultaneously the produced X-rays after having passed a selective absorber either a foil or a gas-filled absorption chamber are detected by a proportional counter or a lithiumdrifted silicon detector $[\mathrm{Si}(\mathrm{Li})]$ followed by electronic devices for pulse height analysis. In the case of lowenergy X-ray photons (say $\lesssim 1 \mathrm{keV}$ ) to be detected a windowless semiconductor device or a flow-type proportional counter is used. The above enumerated main components for measuring yields of X-ray production are standard with the exception of the more or less refined target chamber and low energy X-ray detection devices. A schematic diagram of a typical experimental apparatus as used by $\mathrm{K}$. S. Shima et al. [19] for yield measurements represents figure 4 .

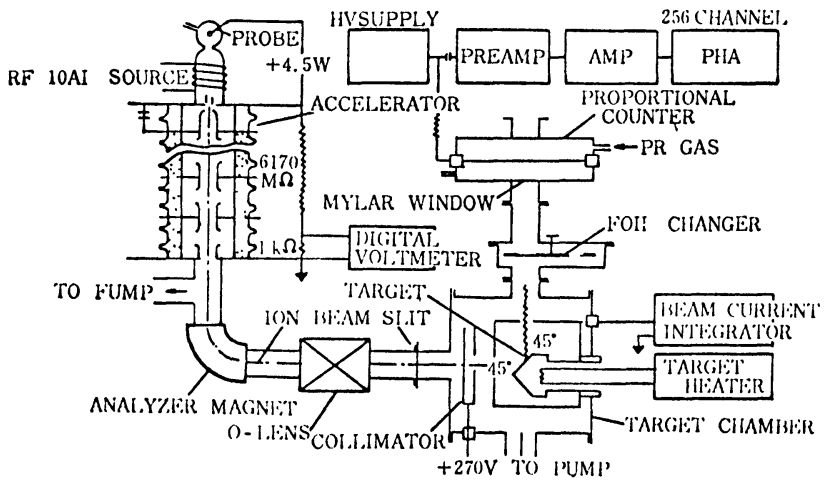

FIG. 4. - Schematic diagram of experimental apparatus used by K. S. Shima et al. [19].

The X-ray yield is extracted from the pulse height distributions of which a typical example is shown in figure 5. This figure reveals the characteristics of the pulse height distribution of proton excited X-rays as compared to the pulse height distribution for electron

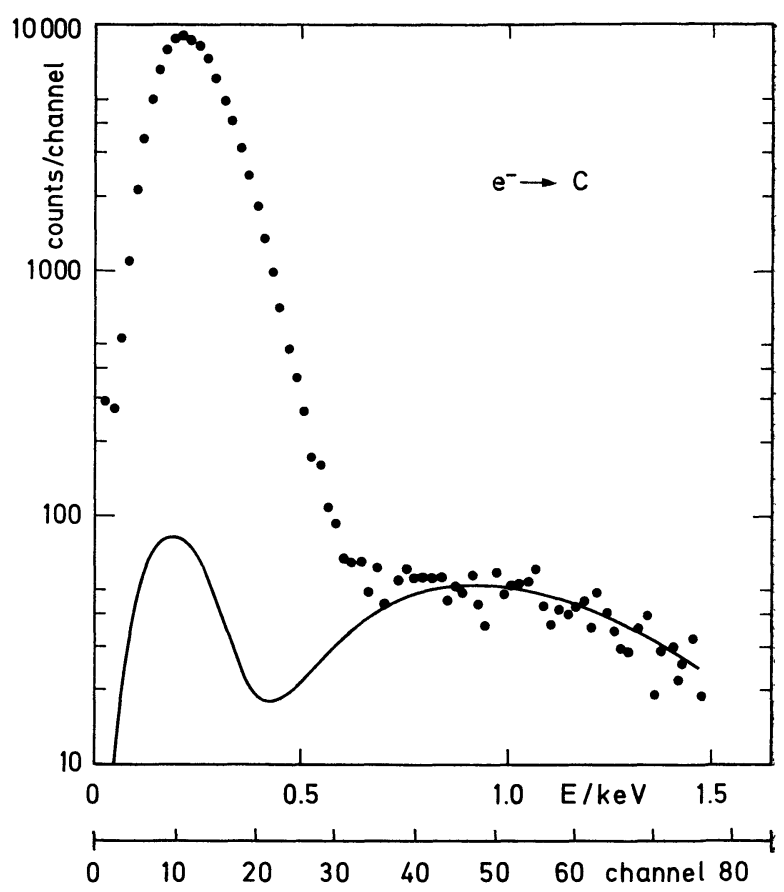

(a)

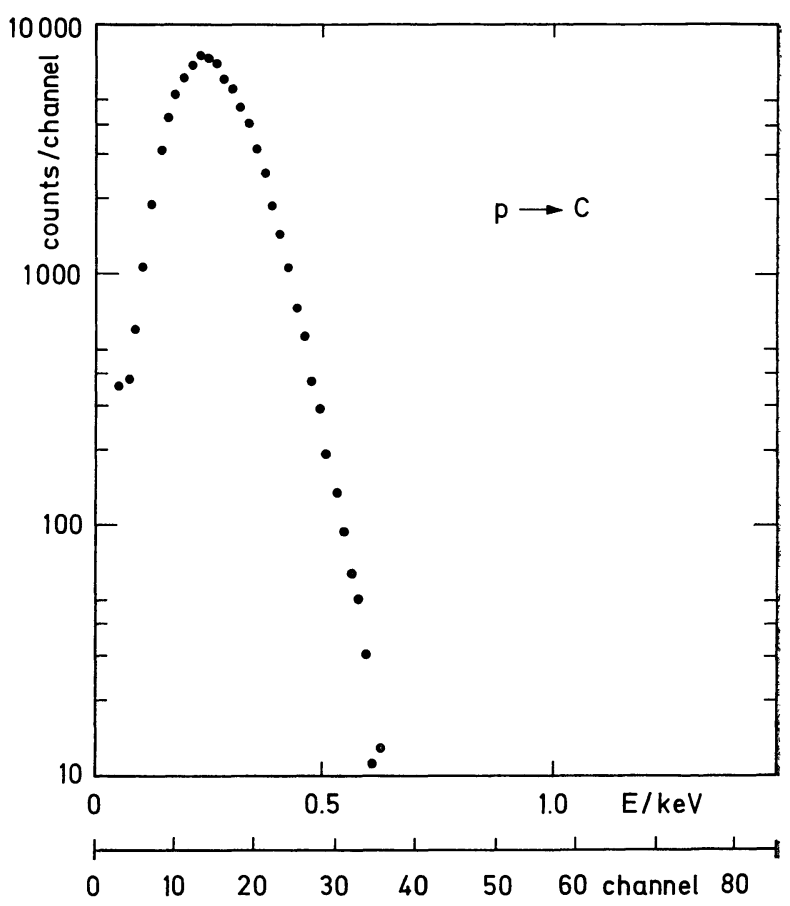

(b)

FIG. 5. - Typical thick target pulse-height distribution for proton-and electron-produced CK-X-rays with a flow proportional-counter. Energies of protons and electrons are $40 \mathrm{keV}$ and $3 \mathrm{keV}$, respectively.

excited X-rays. While the distributions for electrons of a few tens of $\mathrm{keV}$ prominently include a rather complex background due to continuous X-rays, the distributions for protons except at high energies are only influenced by the remaining non-X-ray background, for which correction may be easily made. The more 
serious problem in detecting low-energy-X-rays, such as the $278 \mathrm{eV}$ carbon K-radiation equivalent to $44.5 \AA$, which are of superior importance in applications, e. $g$. in spectrochemical analysis, lies in the electronic noise of the detecting system, which must be kept as small as possible by special precautions. The proper techniques of low-energy X-ray detection are described in detail by Aitken [20]. Moreover, target surface contamination becomes important at low bombarding energies.

Spectral X-ray measurements intended to provide information about the excited state configuration produced in heavy ion-atom collisions, may be performed using the lithium-drifted silicon detector as dispersive element. Usage of Bragg-diffraction technique with its superior resolution $(\approx 1 \mathrm{eV} \mathrm{FWHM})$ as compared to the $\mathrm{Si}(\mathrm{Li})$-detector $(\approx 170 \mathrm{eV} \mathrm{FWHM}$ at $\mathrm{MnK}_{\alpha}$ ) becomes mandatory for X-ray spectra with structures in the $10 \mathrm{eV}$ region. For revealing even finer structures a grazing incidence grating-vacuum X-ray spectrometer has to be applied. As the solid angle of acceptance for the different types of spectrometers goes down as resolution is increased, there may arise counting rate problems which are pronounced if gaseous targets at moderate or low collision energies are to be investigated. These problems may be best handled by automatizing the experimental set-up. In figure 6 an

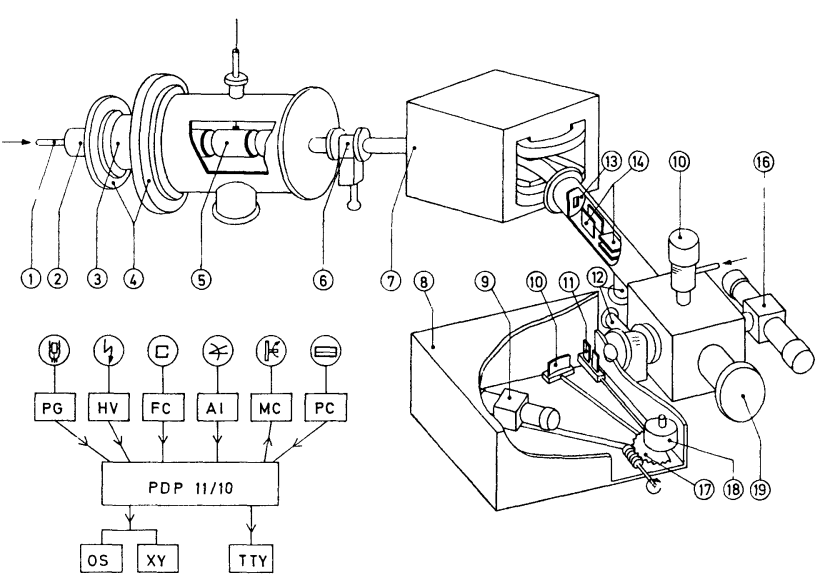

Fig. 6. - Scheme of an experimental arrangement for X-ray yield measurement in solid state or gaseous targets combined with a grating-vacuum-spectrometer for high resolution spectral analysis. 1 : gas inlet ; 2 : ion source ; 3 : extraction electrode ; 4 : isolators ; 5 : electrostatic lens; 6 : valve (separation of beam production and target region); $7:$ mass separation magnet ; 8 : spectrometer chamber ; 9 : proportional counter ; 10 : blazed grating ; 11 : entrance slit ; 12 : outlets for differential pumping of the gas target; 13: mass separation slit; $14:$ deflection plates ; $15:$ target gas inlet ; $16:$ proportional counter ; $17:$ motor drive; $18:$ angular position meter ; 19 : Faraday cup ; PG : pressure gauge ; HV : high voltage (acceleration); FC : Faraday cup ; AI : angular position indicator ; MC : motor control ; PC : pulse counter ; OS : oscilloscope ; XY : XY-writer ; TTY : teletype.

experimental arrangement for X-ray yield measurements in solid state or gaseous targets combined with a grating-vacuum-spectrometer as used in our group is shown.
The experimental aspect of the production of X-rays by ion impact can be thus summarized. Equipment and methods for yield measurements are well established for a wide range of energy and atomic number of projectiles bombarding various targets from carbon to uranium ; this holds also for X-ray spectra with X-ray photon energies down to about $1 \mathrm{keV}$. Below this limit standard equipment and methods begin to fail but just the low photon energy region is by far the most interesting for X-ray analysis.

4. Theoretical background and comparison with experimental results. - Inelastic collisions of ions with atoms leading to excitation and ionization of inner shells, which are manifested directly by the emission of characteristic X-rays and Auger-electrons, have been classified in two broad categories [21]:

1) Collisions, in which the projectile is bare nucleus or acts as a simple charged particle (socalled direct collisions) and

2) slow collisions, in which the projectile carrying an electron cloud forms during the course of the collisions temporarily a quasi-molecule with a target atom due to strong overlapping of the electron clouds of projectile and target atom.

4.1 Production OF INNER SHELL VACANCIES BY direct COLLISIONS. - In ion-atom collisions in which the projectile is a bare charged mass point (e. g. proton, $\alpha$-particle) or in which under given conditions the projectile acts as such only Coulomb excitation of target inner shell electrons can occur. Roughly speaking, the condition to be fulfilled is : Either the atomic number of projectile $Z_{1}$ is much smaller than the atomic number of target atom $Z_{2} . Z_{1} / Z_{2} \ll 1$ is meaning that the projectile $\mathrm{K}$-shell radius $a_{1 \mathrm{~K}}$ is so large, compared to that of the target atom $a_{2 \mathrm{~K}}$, so that projectile K-shell electrons cannot influence the K-shell ionization at any projectile velocity $v_{1}$ or the impact velocity is so high that the projectile electron cloud will be stripped off in collisions with target electrons (the approximate criterium for complete stripping is $\left.v_{1}>v_{2 \mathrm{~K}}\right) ; v_{1}$ and $v_{2 \mathrm{~K}}$ are respectively projectile velocity and characteristic electron velocity in the projectile K-shell $[22,23]$.

The mechanism of Coulomb-excited inner shell vacancy production is described as a direct interaction between the swiftly moving incident charged particle and a tightly bound atomic electron. The Coulomb interaction provides the perturbation that causes transitions between the unperturbed levels of the target atom. The general features of direct Coulomb interaction and ionization by incident heavy charge particles are now well understood as discussed in the review paper by J. D. Garcia, R. J. Fortner and T. H. Kavanagh [13] and by E. Merzbacher [21]. Within this frame predictions of cross-sections for inner shell ionization have been made in different approaches of which the Plane Wave Born Approximation (PWBA) in the extreme form $[1,24]$ or in refined form $[22,25]$ 
ncluding Coulomb deflection and binding effects is usually refered to.

In the impulse approximation the dominant interaction producing the transitions is supposed to be a direct energy exchange between the incident charged particle and the bound electron. A widely used formulation within the impulse approximation is the Binary Encounter Approximation (BEA) [26].

The computations of the total cross-section for $\mathrm{K}$-shell ionization $\sigma_{\mathrm{K}}^{\mathrm{PWBA}}$ in the PWBA approach using screened non-relativistic hydrogenic wave functions (using wave functions in the Hartree-Slater approach does not give significantly different results [25]) lead to the expression

$$
\begin{gathered}
\sigma_{\mathrm{K}}^{\mathrm{PWBA}}=\sigma_{0 \mathrm{~K}} \eta_{\mathrm{K}}^{-1} f_{\mathrm{K}}\left(\eta_{\mathrm{K} ;} ; \theta_{\mathrm{K}}\right) \\
\sigma_{0 \mathrm{~K}}=\left(\frac{Z_{1}}{Z_{2 \mathrm{~K}}}\right)^{2} \cdot 8 \pi\left(\frac{a_{0}}{Z_{2 \mathrm{~K}}}\right)^{2}:
\end{gathered}
$$

suitable unit for the scaling of a $\mathrm{K}$-shell radius ; $a_{0}$ : Bohr radius; $Z_{1}$ : atomic number of projectile ; $Z_{2 \mathrm{~K}}=Z_{2}-0.3$ : effective atomic number of target atom.

$$
\eta_{\mathrm{K}}=\frac{m_{\mathrm{e}}}{M_{1}} \cdot \frac{E}{Z_{2 \mathrm{~K}}^{2}} \frac{E}{\mathrm{Ry}} \equiv\left(\frac{v_{1}}{v_{2 \mathrm{~K}}}\right)^{2}:
$$

reduced projectile velocity which is proportional to its initial kinetic energy $E ; v_{1}$ : projectile velocity; $v_{2 \mathrm{~K}}$ : characteristic electron velocity in the target atom K-shell ; Ry : 1 Rydberg $=13.6 \mathrm{eV} ; m_{\mathrm{e}}$ and $M_{1}$ : electron respectively projectile mass.

$$
\theta_{\mathrm{K}}=\frac{u_{\mathrm{K}}}{Z_{2 \mathrm{~K}}^{2} \cdot \mathrm{Ry}}:
$$

$K$-shell screening number $\equiv$ ratio of the observed binding energy $u_{\mathrm{K}}$ in the $\mathrm{K}$-shell of the target atom to its hydrogenic value.

Graphs [1] and tables [24] of the dimensionless function $f_{\mathrm{K}}\left(\eta_{\mathrm{K}} ; \theta_{\mathrm{K}}\right)$ have been published for the K-shell (also the functions $f_{\mathrm{L}}, f_{\mathrm{L}_{\mathrm{I}}}$ and $f_{\mathrm{L}_{\mathrm{II}}}$ ) so that $\sigma^{\mathrm{PWBA}}$ for the $\mathrm{K}$ - and L-shell as well as for the L-subshells in this extreme may be predicted.

Since $\theta_{\mathrm{K}}$ appears in the function $f_{\mathrm{K}}\left(\eta_{\mathrm{K}} ; \theta_{\mathrm{K}}\right)$ approximately only in combination with $\eta_{\mathrm{K}}$ as a universal variable $\eta_{\mathrm{K}} / \theta_{\mathrm{K}}^{2}$ and as an independent parameter, one may reduce the cross sections as done by G. Basbas, W. Brandt and R. Laubert [25] by virtue of the approximate relation which becomes inaccurate for $\eta_{\mathrm{K}}>1[1]$

to

$$
\begin{aligned}
& f_{\mathbf{K}}\left(\eta_{\mathbf{K}} ; \theta_{\mathbf{K}}\right) \cong \theta_{\mathbf{K}} f_{\mathbf{K}}\left(\eta_{\mathbf{K}} / \theta_{\mathbf{K}}^{2} ; 1\right) \\
& \frac{\sigma_{\mathbf{K}}^{\text {PWBA }}\left(\eta_{\mathbf{K}} ; \theta_{\mathbf{K}}\right)}{\sigma_{0 \mathbf{K}} / \theta_{\mathbf{K}}}=F\left(\eta_{\mathbf{K}} / \theta_{\mathbf{K}}^{2}\right)
\end{aligned}
$$

This reduced ionization cross-section depends only on the variable $\eta_{\mathrm{K}} / \theta_{\mathrm{K}}^{2}$. The above mentioned authors [25] tabulated the universal function $F\left(\eta_{\mathrm{K}} / \theta_{\mathrm{K}}^{2}\right)$. Their results mean that within the range of a valid application of the PWBA-approach all cross-sections reduced according to the prescription should fall on a universal curve in such a scaled plot. The range of valid application (supposed to have a lower limit at $\eta_{\mathrm{K}}=0.015$ [24]) requires for the projectile small charge $\left(Z_{1} \ll Z_{2}\right)$ and a high enough velocity of the projectile to ensure a negligible effect of nuclear Coulomb deflection.

Testing this assertion by plotting in this reduced scale experimental data for different projectiles impinging at various energies on different targets reveals systematic deviations from the calculated PWBA values. Experimental cross-sections fall short of the predicted ones growingly with decreasing $\eta_{\mathrm{K}} / \theta_{\mathrm{K}}^{2}$ as shown in figure 7 taken from [25] and can exceed one order of magnitude.

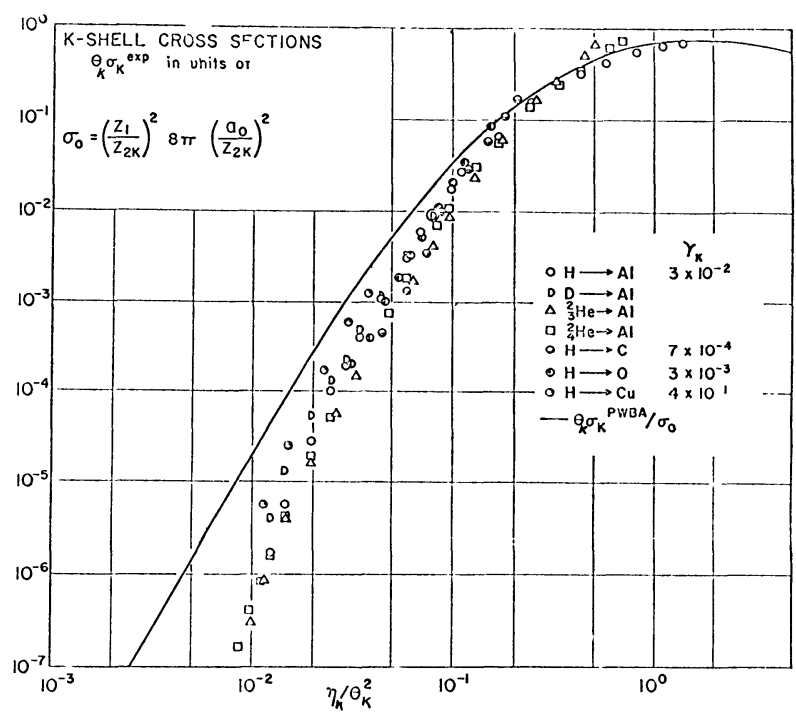

FiG. 7. - Reduced experimental K-shell cross-sections as function of a reduced projectile velocity variable. The extreme PWBA-model predicts that all data should fall on the fully drawn curve, the universal function $F\left(\eta_{\mathrm{K}} / \theta_{\mathrm{K}}^{2}\right)$ [25]. $\gamma_{\mathrm{K}}$ is the fluorescence yield.

Refinements of the PWBA approach lead to taking into account the deflection of the projectile in the Coulomb field of the target nucleus, as done by J. Bang and J. M. Hansteen [27] in their semi-classical treatment of $\mathrm{K}$-shell ionization cross-sections, and the increased binding of the atomic electrons to the impinging proton as proposed by W. Brandt, R. Laubert and E. Sellin [28]. If the binding effect is taken approximately into account by a binding factor $\varepsilon=\varepsilon\left(\eta_{\mathrm{K}}\right)$ applied to $\theta_{\mathbf{K}}$ and the Coulomb deflection by a Coulomb deflection factor $9 E_{10}\left(\pi d q_{0} \varepsilon\right)$ applied to $\sigma_{\mathrm{K}}^{\mathrm{PWBA}}$, where $E_{10}$ : exponential integral of order $10 ; d$ : half distance of closest approach $; \hbar q_{0}:$ minimum momentum transfer, $\sigma_{\mathrm{K}}$ becomes

$$
\begin{aligned}
\sigma_{\mathrm{K}} & =\sigma_{0 \mathrm{~K}} 9 E_{10}\left(\pi d q_{0} \varepsilon\right) \eta_{\mathrm{K}}^{-1} f_{\mathrm{K}}\left(\eta_{\mathrm{K}} ; \varepsilon \theta_{\mathrm{K}}\right) \\
& \cong \sigma_{0 \mathrm{~K}} \cdot \frac{9 E_{10}\left(\pi d q_{0} \varepsilon\right)}{\varepsilon \theta_{\mathrm{K}}} \cdot F\left(\eta_{\mathrm{K}} /\left(\varepsilon \theta_{\mathrm{K}}\right)^{2}\right)
\end{aligned}
$$


and the systematic deviations as is demonstrated in figure $8[25]$ are removed up to $\eta_{\mathrm{K}} /\left(\varepsilon \theta_{\mathrm{K}}^{2}\right) \approx 0.05$. The remaining deviations in this region may possibly be traced back to the accuracy of experimental data and to

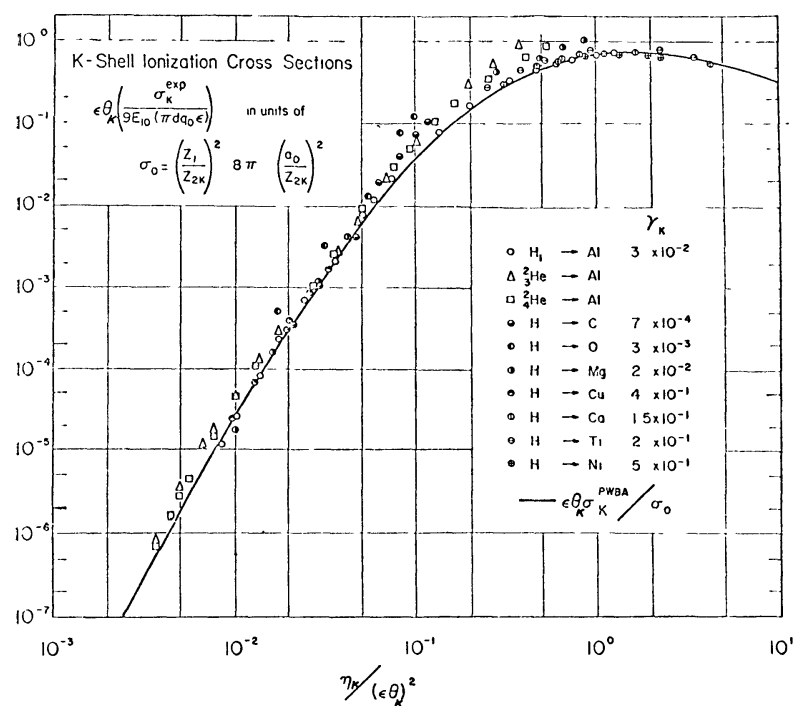

Fig. 8. - Reduced experimental K-shell cross-sections as function of a reduced projectile velocity variable. Applying factor in the extreme PWBA-calculations to account for Coulomb-deflection and binding the data fall in good approximation on the fully drawn curve, the universal function $F\left(\eta_{\mathrm{K}} / \theta_{\mathrm{K}}^{2}\right)[25]$.

variation in data analysis ; especially the problem of the proper choice of fluorescence yield in converting $\mathrm{X}$-ray data to cross-section is always present for low atomic number targets. The discrepancies for $\eta_{\mathrm{K}} /\left(\varepsilon \theta_{\mathrm{K}}^{2}\right)>0.05$ (high collision energies) are discussed by G. Basbas, W. Brandt and R. Laubert [25].

The approach by J. D. Garcia [29] (see ref. [26]), an almost completely classical model known as the classical binary encounter approximation (BEA) provides also a very satisfactory formula for the $\mathrm{K}$-shell ionization cross-sections as function of energy for light ions. In this BEA-model the ionizing collision is treated as a collision between the incident projectile and a free electron whose velocity follows a probability distribution characteristic of the initial atomic state. To good approximation this model suggests that the quantity $u_{\mathrm{K}}^{2} \cdot \sigma_{\mathrm{K}}^{\mathrm{BEA}} / Z_{1}^{2}$ should be a universal function of $E / \lambda u_{\mathrm{K}}$, the ratio of the incident particle energy to the binding energy of the atomic electron in the target times $\lambda\left(\lambda\right.$ is the mass $M_{1}$ of the projectile in electron mass units, $\lambda=M_{1} / m_{\mathrm{e}}$ ). Otherwise, this quantity is independent of target atom parameters. A comparison (Fig. 9) with experimental data for proton and $\alpha$-particle impact confirms this prediction [30].

A general comparison of the PWBA- and BEAapproaches with experimental cross-sections by proton impact has been presented by J. D. Garcia et al. (Fig. 10) [13]. All the data except for the $160 \mathrm{MeV}$ points form a reasonably compact curve above

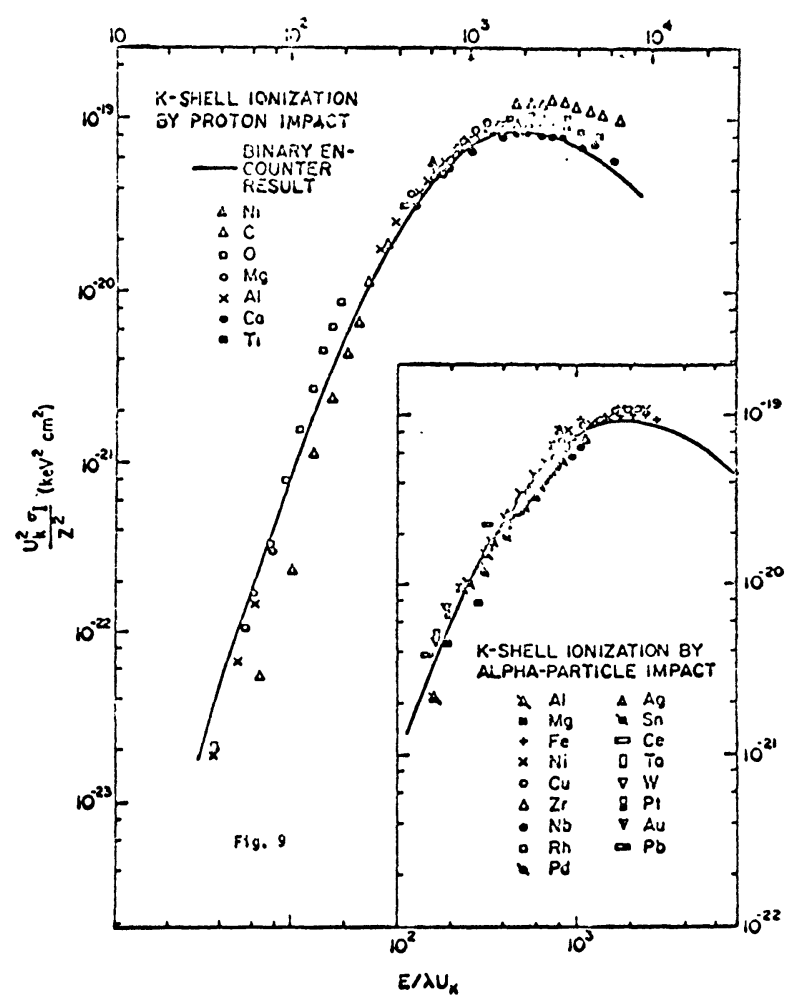

FIG. 9. - K-shell ionization cross-sections for protons and $\alpha$-particles [30].

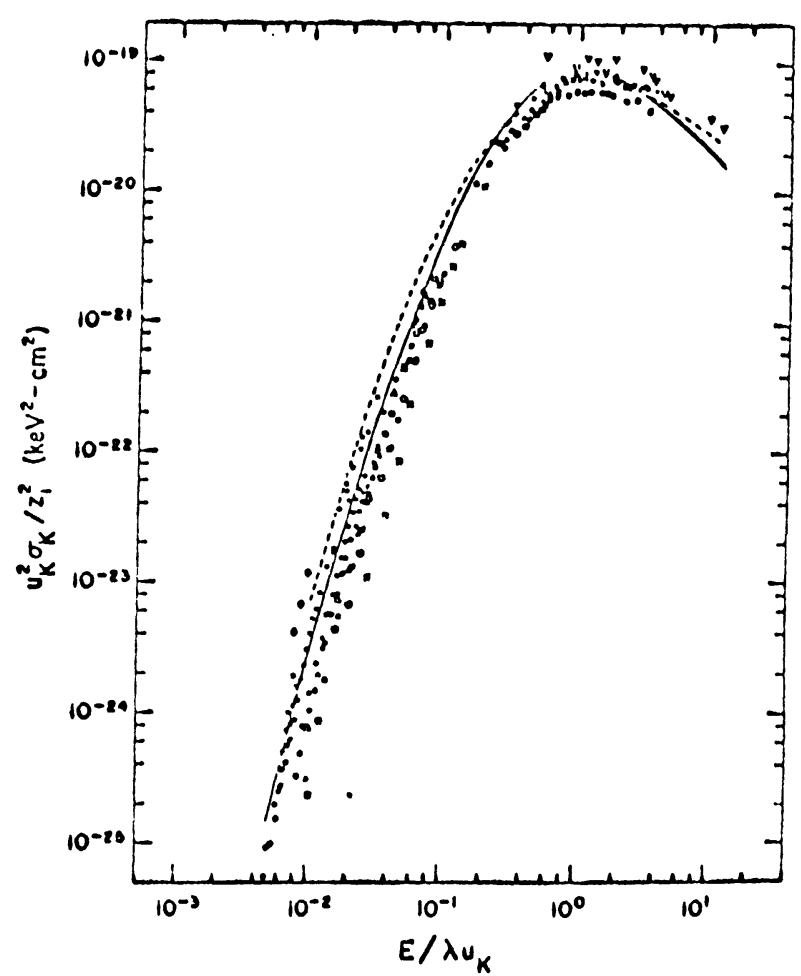

Fig. 10. - General comparison of PWBA- and BEA-predictions with experimental data for proton impact : dashed curve, extreme PWBA; solid curve, BEA; experimental data for various target elements [13].

$E / \lambda u_{\mathrm{K}}>10^{-1}$. Below, the data for targets with $15<Z_{2}<70$ continue to define a universal function, but the data for low atomic number elements begin to 
spread in the direction of smaller cross-sections. To reveal characteristics for low atomic number elements, most of the known data for carbon are presented in figure 11 .

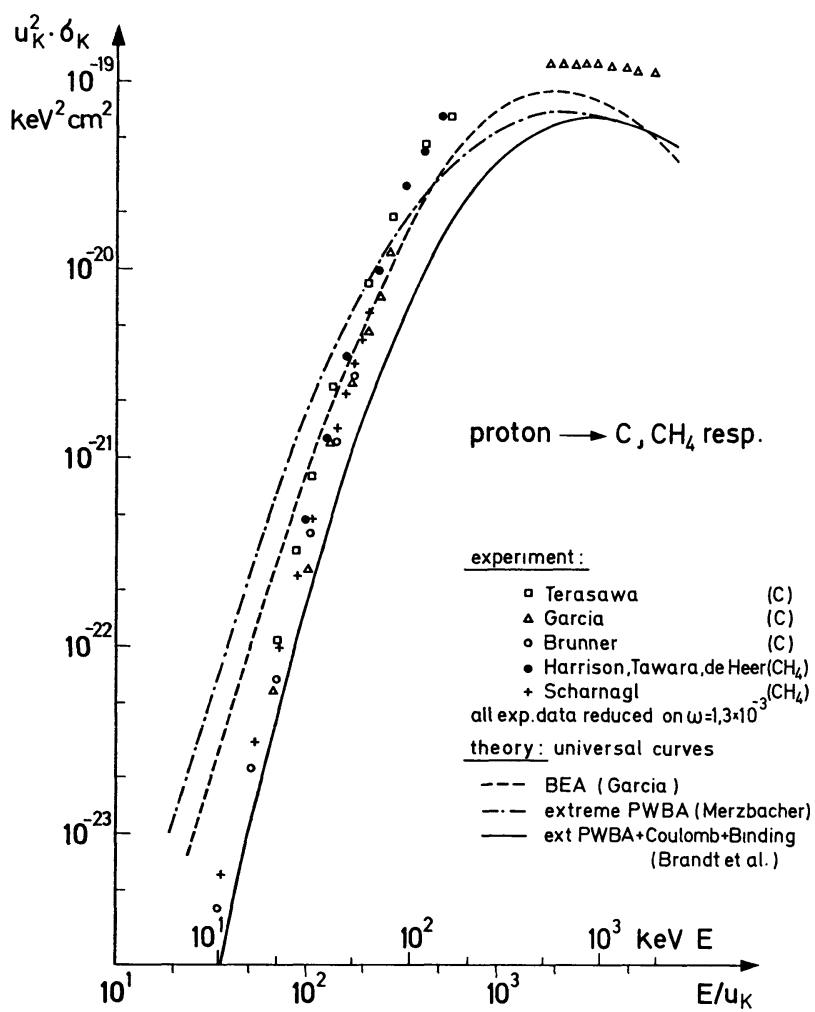

Fig. 11. - Theoretical and experimental carbon K-shell ionization cross-sections for proton impact.

4.2 Production of INNer SHell Vacancy BY COLLISIONS OF HEAVY IONS. - Slow collisions where the projectiles with atomic number comparable to that of the target atoms $\left(Z_{\mathrm{proj}} \approx Z_{\mathrm{targ}}\right)$ carry an electron cloud of comparable size into the encounter such that strong overlapping of electron clouds occur and, roughly speaking, new effects due to the exclusion principle arise. These lead to a further mechanism of inner shell vacancy production with high cross-sections for even very slow collisions as is examplified in figure 12 [13], in which the carbon $\mathrm{K}$-shell ionization cross-section as function of the kinetic energy per mass unit of the different projectiles is shown. In the nonrelativistic region $E / M_{1}$ is proportional to the projectile velocity $v_{1}$ squared, thus cross-sections for different projectiles at the same projectile velocity are compared in the figure. It can be seen that the cross-sections for heavy ion impact are several orders of magnitude greater than that for protens of the same velocity; but even for the same kinetic energy of the proton the cross-sections are comparable or greater. The increased efficiency of L-shell vacancy production by heavy ion impact has been observed also, for instance by F. W. Saris [31], who measured the ArL-X-ray crosssection in Ar gas targets for various incident heavy ions

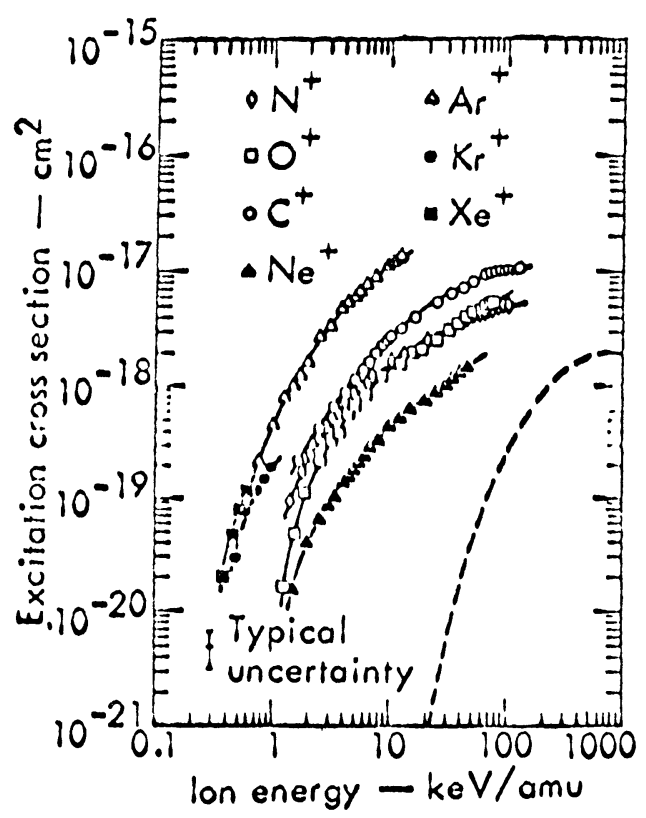

FIG. 12. - Experimental carbon K-shell excitation crosssections as a function of incident ion energy per mass unit for various heavy ions. The dashed curve is for incident protons [13].

(Fig. 13) ; moreover, from these measurements it can be seen that cross-sections drop to a sharp thresholdlas the projectile energy is lowered. A more general demonstration that Coulomb excitation cannot account

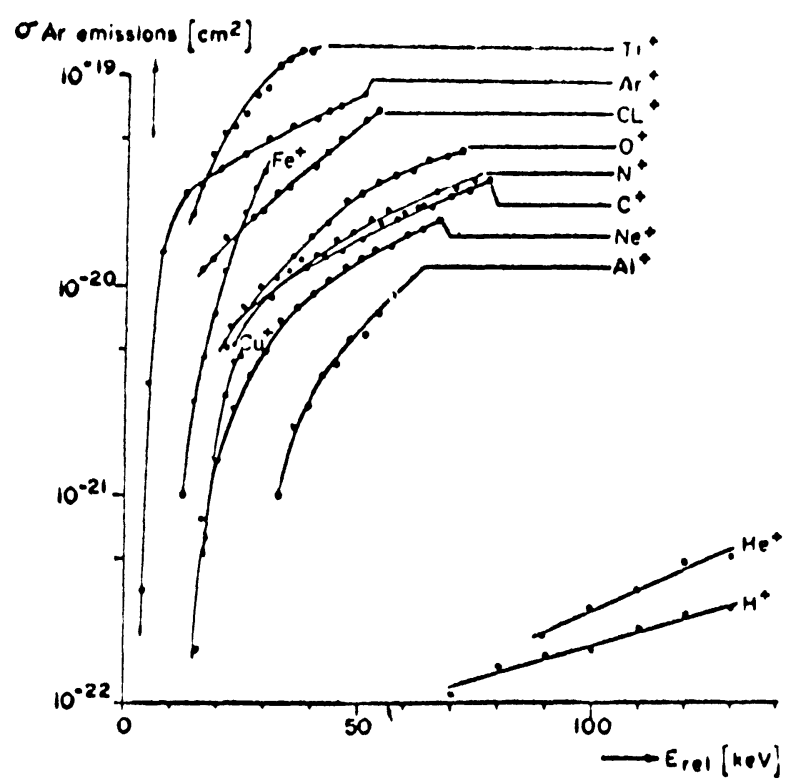

Fig. 13. - Experimental ArL X-ray production cross-section in Ar gas target for various incident heavy ions [31].

for the high cross-sections at low velocity is given if one includes experimental cross-sections for $Z_{\mathrm{proj}} \approx Z_{\mathrm{targ}}$ on the universal graph scaled for Coulomb excitation (Fig. 14) as has been done by W. Brandt [22]

The new mechanism referred to is that of electron promotion in the molecular orbital model the applica- 


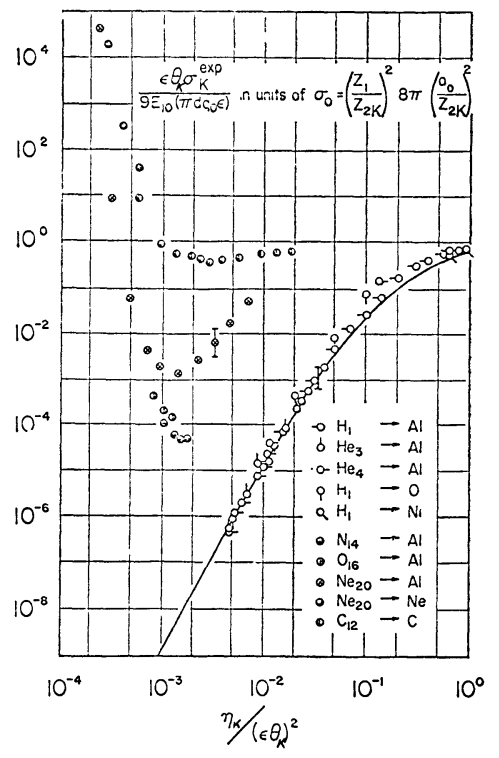

FIG. 14. - Experimental K-shell ionization cross-section of various targets for heavy ion impact in a reduced plot used in figure 7 and 8 [22].

tion of which to inner shell vacancy production in heavy ion-atom collisions was first suggested by U. Fano and W. Lichten [11] in their interpretation of L-shell excitation in $\mathrm{Ar}^{+}-\mathrm{Ar}$ collisions, further developed by W. Lichten [32] and reviewed recently by M. Barat and W. Lichten [33].

The starting point for a rigorous theoretical description in such inelastic collisions is [13] : If the relative projectile velocity $v_{1 \mathrm{CM}}$ is small compared to the characteristic velocity $v_{2 \mathrm{~K}}$ of a target $\mathrm{K}$-shell electron.

$v_{1 \mathrm{CM}}$ : projectile velocity in the counter of mass system ;

$$
\left(\frac{v_{1 \mathrm{CM}}}{v_{2 \mathrm{~K}}}\right)^{2} \equiv \frac{E_{\mathrm{CM}}}{\lambda u_{\mathrm{K}}} \ll 1
$$

$v_{2 \mathrm{~K}}$ : characteristic velocity of the target $\mathrm{K}$-shell electrons ;

$E_{\mathrm{CM}}$ : initial kinetic energy of the projectile in the CM-system ;

$\lambda: \mu / m_{\mathrm{e}}$ with $\mu$ reduced mass of projectile (under this condition the electronic wave functions may strongly overlap), the K-shell electrons adjust nearly adiabatically to the second force center and a transient diatomic molecule is formed during the collision. Drastic changes occur in the electronic level structure by the formation of this quasi-molecule, the electrons going into various corresponding molecular orbitals with orbital energies to be calculated in the Born-Oppenheimer approximation depending parametrically on the internuclear distance. Then upon separation of the nuclei in the further course of the collision, the electrons will go back to the initial state. But due to the small perturbation of the stationary electronic states of the quasi-molecule by the motion of the nuclei, transitions may be caused so that an electron may find itself promoted in a higher electronic state of the atom upon separation. If this happens for an inner shell electron, the atom will end and up with an inner shell vacancy.

Within this frame J. S. Briggs and J. Macek [34] estimated the cross-section for $\mathrm{K}$-shell vacancy production in $\mathrm{Ne}^{+}-\mathrm{Ne}$ collisions and J. S. Briggs [35] for $\mathrm{C}^{+}-\mathrm{C}, \mathrm{N}^{+}-\mathrm{N}, \mathrm{O}^{+}-\mathrm{O}$ collisions in an ab initio treatment of interactions in which rotational coupling provides the excitation mechanism. A less rigorous approach is used by R. C. Fortner et al. [36] for the excitation of cross-sections. The starting point is the Fano-Lichtenmodel [11]. In this model one-electron molecular diabatic states are constructed at all values of internuclear separation by approximating the molecular wave functions as combination of hydrogenic one-electron orbitals, thus neglecting configuration interaction. The representation of the resulting orbital energies (with nuclear repulsion removed) as function of internuclear separation (called correlation diagram ; the construction of a correlation diagram and its consequences for electron promotion have been comprehensively reviewed by M. Barat and W. Lichten [33]) show diabatic crossings at various internuclear separations (Fig. 15) [37]. The model states the correlation rules

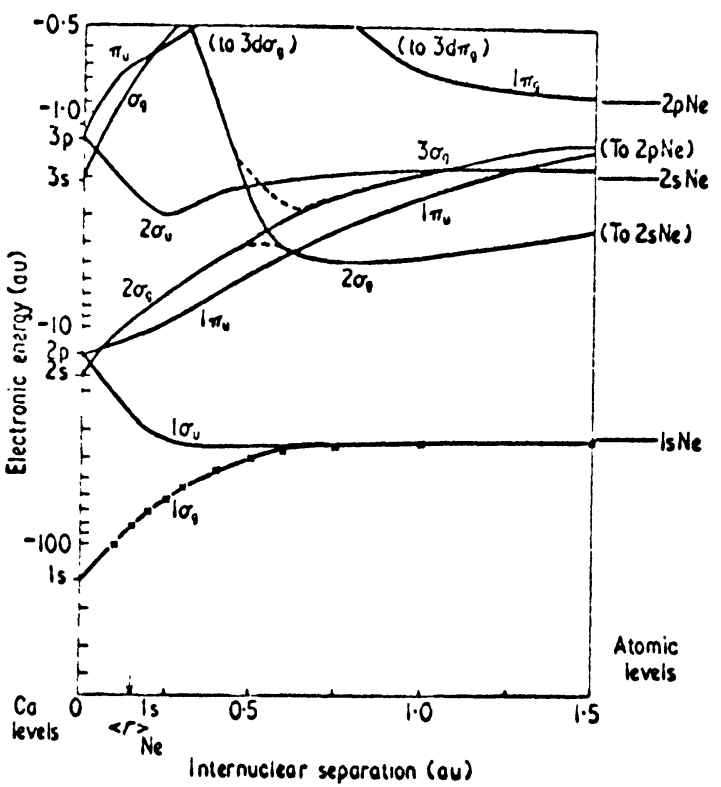

Fig. 15. - Correlation diagram for the Ne-Ne-system based upon ab initio molecular orbital calculation. Near the crossing of diabatic curves (fully drawn) the computed adiabatic curves at internuclear distances marked by crosses are broken [37].

which prescribe the correlation of the separated and united atom-states via the molecular orbitals. It predicts that at the crossing of an initially filled with an unfilled orbital the probability for an electronic transition between these orbitals is highly peaked. Applying the Landau-Zener theory of level crossing (see N. F. Mott and S. N. Massey [38]), which gives the probability for such a transition, the cross-section for vacancy production can be estimated as was done by R. J. Fortner et al. [36]. 
The approach of Q. C. Kessel [39] relies on an empirically based assumption for the dependence of the transition-probability on the impact parameter.

The predicted cross-sections for $\mathrm{K}$-shell ionization in these different approaches are compared with experimental data in figure 16. The agreement with the rigorous Briggs- and Macek-approach is very encouraging, and more work on this line seems to be promising (for a detailed discussion see [13]).

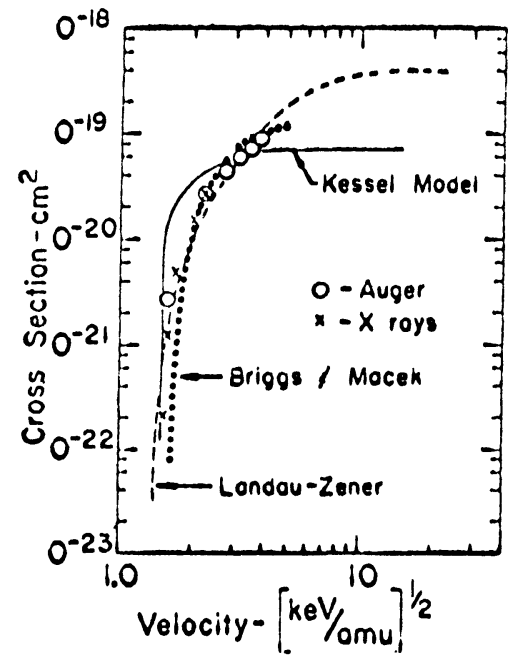

FIG. 16. - Theoretical and experimental cross-sections for $\mathrm{K}$-shell excitation in $\mathrm{Ne}^{+}-\mathrm{Ne}$-collisions as function of the incident ion velocity [13].

5. Experimental results of relevance for applications. - Under suitably chosen conditions ion-produced X-rays might turn out to become one of the most sensitive analytic technique that is available in modern technology. Therefore some of the experimental results which lay the foundations for this possible application will be given first.

Many authors have reported results on the production of K-, L-, M- and N-shell X-rays excited in various targets by ion impact. U. J. Ling et al. [40] have published a table which enumerates exhaustively the work on ionization cross-sections up to 1971, for more recent work see reference [2]. A table of X-ray production cross-section measurements for $p, d, \alpha$-particle impact is given in [13].

As to proton-produced X-rays the targets vary from Be to $U$ at impact energies ranging from a few $\mathrm{keV}$ up to several MeV. From the published cross-section data the X-ray yield can easily be evaluated for different target conditions. As examples of these data thick target yields as a function of incident proton energy (100$300 \mathrm{keV}$ ) measured by K. S. Shima et al. [19] are shown in figure 17. The authors estimated to about $\pm 5 \%$ as is usual in yield measurements. These authors present their data in a log-log plot thus revealing the approximate validity of a power law $Y=a \cdot E^{b}$ for the yield in the limited range of energy used, where $a$ and $b$ are constants depending on target material. The power $b$

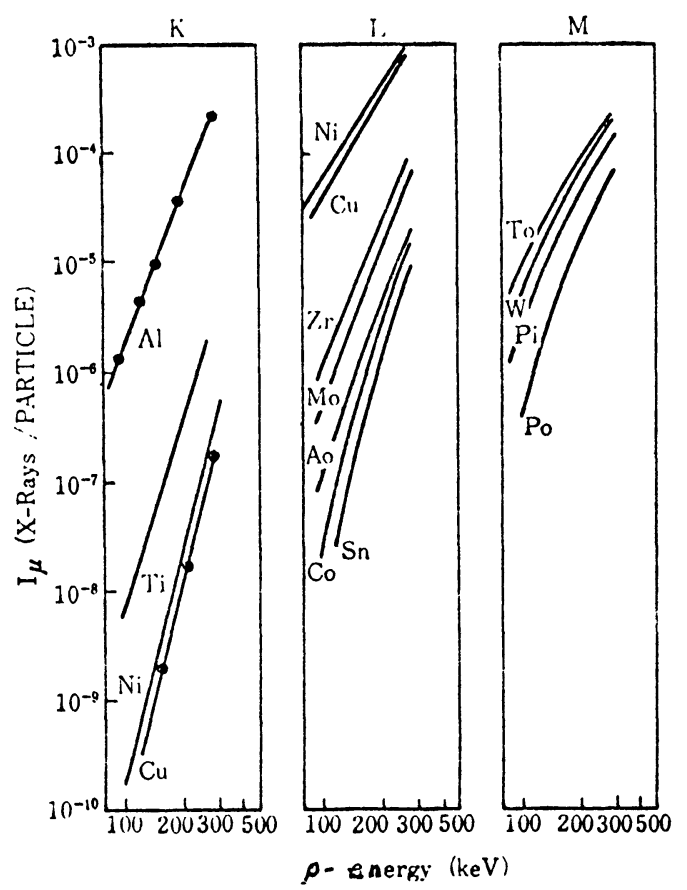

FIG. 17. - Experimental thick target yield $I_{\mu}$ of K-, L- and M-X-rays for proton impact [19].

ranges from $4.6(\mathrm{AlK})$ to $8.0(\mathrm{CuK})$, from $3.0(\mathrm{NiL})$ to $6.2(\mathrm{SnK})$, and from 3.6 (TaM) to 4.7 (PbM). Generally such a power law must fail as the yield-versus-energy curve is peaked in the MeV-region as predicted from cross-sections and proved experimentally for instance by G. A. Bissinger [41]. The strong dependence on the target atomic number $Z$ is displayed in figure 18 [42]

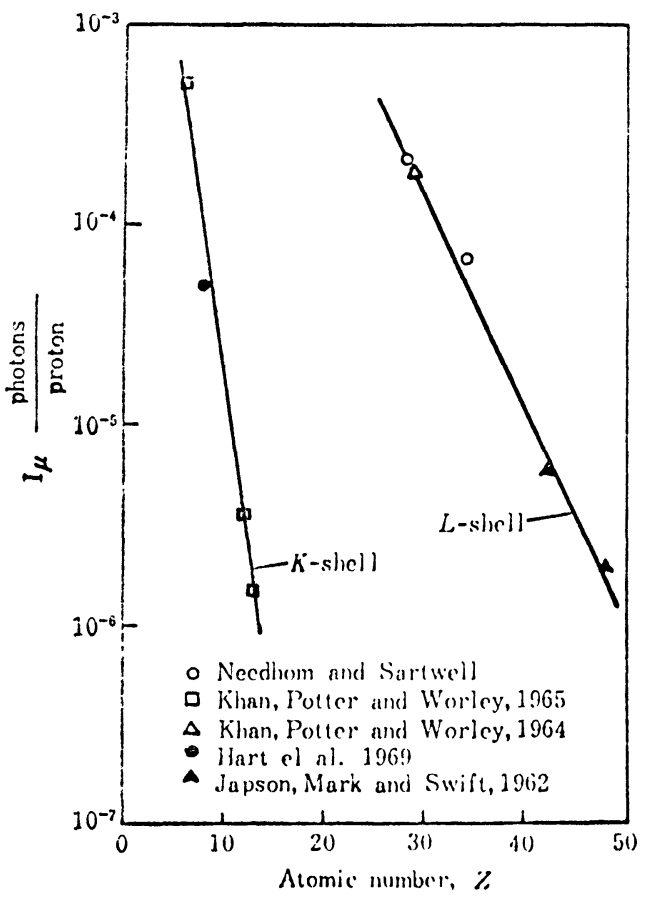

FIG. 18. - Dependence of experimental thick target X-ray yield $\mathbf{I}_{\mu}$ on the target atomic number $Z$ at fixed proton energy of $100 \mathrm{keV}$ [42] 
which combines measurements of various authors at fixed proton energy of $100 \mathrm{keV}$. An interesting feature is revealed if the yield for fixed proton energy is plotted as function of the wave length of the produced X-rays (Fig. 19) [43]. The X-ray yield is somewhat higher for transitions between higher shells.

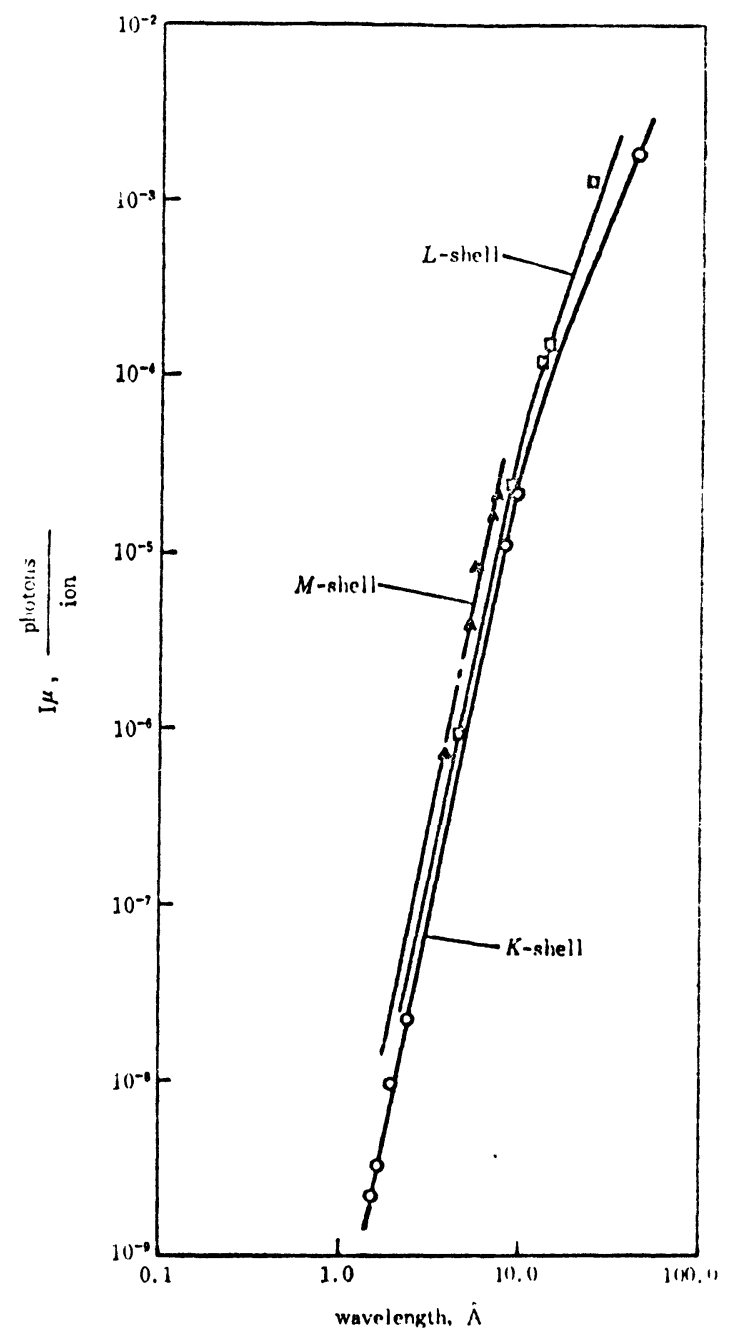

Fig. 19. - Dependence of experimental thick target yield on the wavelength of emitted X-rays excited by $150 \mathrm{keV}$ protons [43].

The recent experiments and theoretical work on heavy ion-produced X-rays (see ref. $[12,13]$ ) have clearly demonstrated the different mechanisms. The consequences of this fact lead to some important distinctions between heavy ion- and proton-induced $\mathrm{X}$-rays.

5.1 In high resolution experiments satellite spectral lines are observed (Fig. 20) [44] due to transitions in multiply ionized atoms [45-49]. One practical consequence is that the $\mathrm{X}$-ray line under study may occupy a larger proportion of the spectrum than anticipated as shown in figure 21 [50] by a comparison of $\mathrm{e}^{-}, \mathrm{H}^{+}$and $\mathrm{Ne}^{+}$produced CoL X-rays and could cause problems in separating out various transitions. However, by this

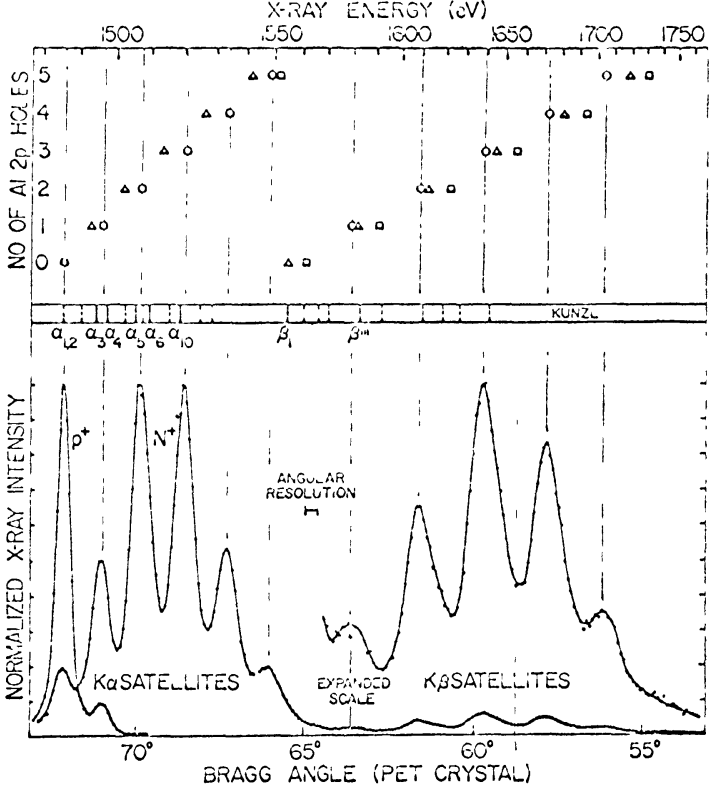

FIG. 20. - Aluminum K-X-ray spectrum excited by $5 \mathrm{MeV}$ protons and nitrogen ions. At the top the measured peak energies (crosses) are compared with calculations assuming $2 p$ vacancies in addition to the 1s vacancy [44].

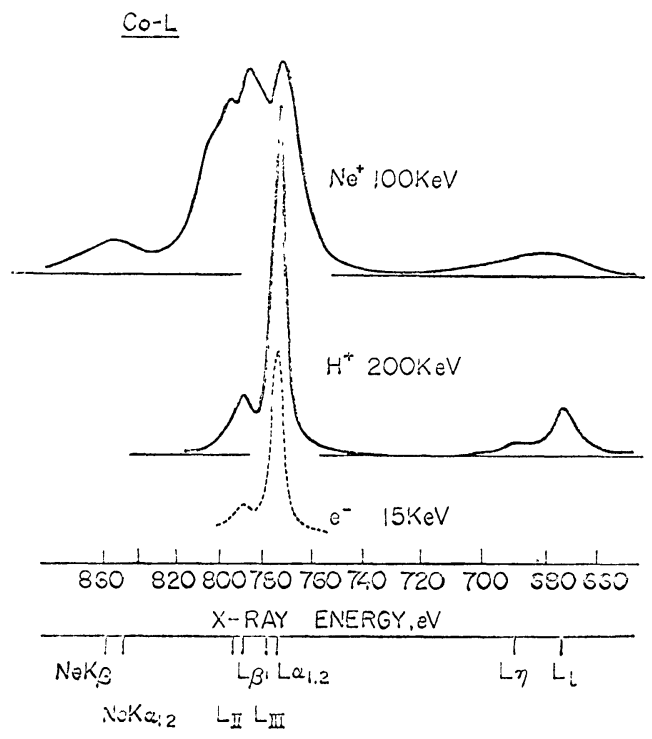

Fig. 21. - Experimental CoL X-ray spectra excited by $\mathrm{e}^{-}$, $\mathrm{H}^{+}$and $\mathrm{N}^{+}$impact respectively [50].

kind of energy broadening not necessarily analytical difficulties arise, for example it is easy to separate out the $\mathrm{K}_{\alpha}$-lines of the adjacent elements $\mathrm{Al}$ and $\mathrm{Si}$, using a crystal spectrometer, even in the presence of their satellites [51].

5.2 Recently there have been cited several examples of X-rays which cannot be assigned to transitions within either the target or the projectile (for newer data see ref. $[15,53])$. Such X-rays have been described by F. W. Saris et al. [59] as arising during the life time of the quasi-molecule formed in the course of the 
collision. Figure 22 gives an example for such continuous molecular X-rays produced by Ar bombardment of Ti. A band of several $100 \mathrm{eV}$ width is observed [53].

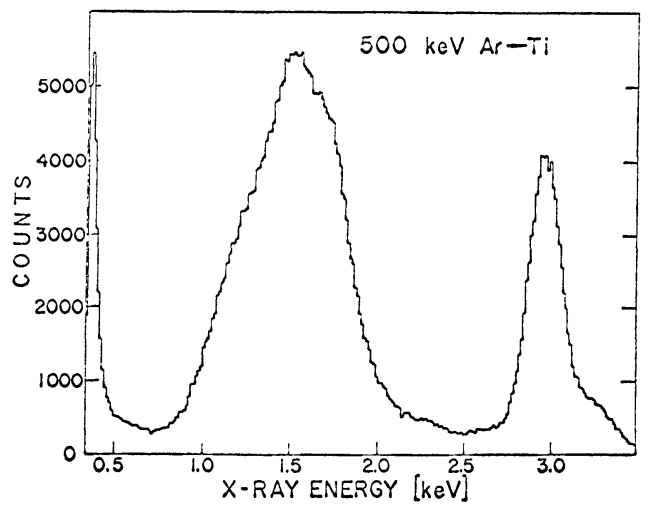

Fig. 22. - Experimental X-ray spectrum produced by $500 \mathrm{keV}$ $\mathrm{Ar}^{+}$impact on $\mathrm{Ti}$. A broad X-ray distribution (molecular $\mathrm{X}$-rays) is observed along with the $\operatorname{ArK}_{\alpha}$-line (2.96 keV) [53].

To explain the occurance of such molecular X-rays a twofold collision process is assumed. In the first collision an L-shell vacancy is produced, within the life time of the vacancy a second collision has to take place in the course of which the vacancy in the quasi-molecule decays and a molecular X-ray photon may be emitted. The molecular X-rays will form a broad band because the transition energy between the molecular orbitals will continuously change due to the change of internuclear separation during the collision. With growing impact energy the band will change towards the characteristic X-ray energy of the united atom.

The molecular $\mathrm{X}$-rays will act as a continuous background in X-ray spectra and thus may limit the sensitivity in analytical applications.

5.3 The fluorescence yield $\omega$ has been taken to be a definite quantity for each subshell of each atomic species. This implies that the de-excitation process is independent of the mode by which the excitation took place. But by comparison of L X-ray cross-sections in $\mathrm{Ar}^{+}$-Ar collisions with Auger-electrons cross-sections it was found that $\omega_{\mathrm{L}}$ increases by an order of magnitude as the collision energy goes from $8-15 \mathrm{keV}$ and then changes more slowly (Fig. 23, [9]). In 0.3 to $2.0 \mathrm{MeV}$ proton collisions with carbon L. H. Toburen [54] observed a similar variation of the apparent fluorescence yield with energy comparing Auger-electron yields with published X-ray yields.

F. P. Larkins [55] has shown that these experimental results can be explained in terms of multiple vacancy production in the collisions, requiring that the fluorescence yield has to be defined for each state associated with a vacancy. Using this model several calculations of the fluorescence yield for various vacancy configurations have been done [56-62] (Fig. 24). From the present analysis of fluorescence yield data follows clearly that the use of a constant value for $\omega$ in the analysis

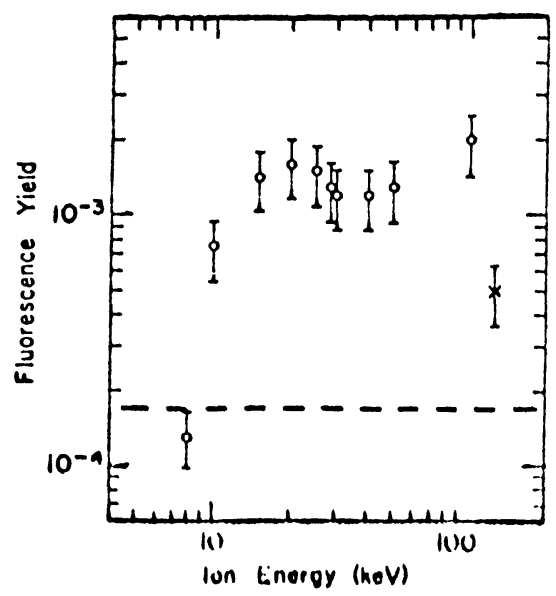

Fig. 23. - ArL-shell fluorescence yield in $\mathrm{Ar}^{+}$-Ar collisions as a function of ion energy from measured data. The cross represents a value for $125 \mathrm{keV}$ proton impact and the dashed line a theoretical value for the neutral atom [9].

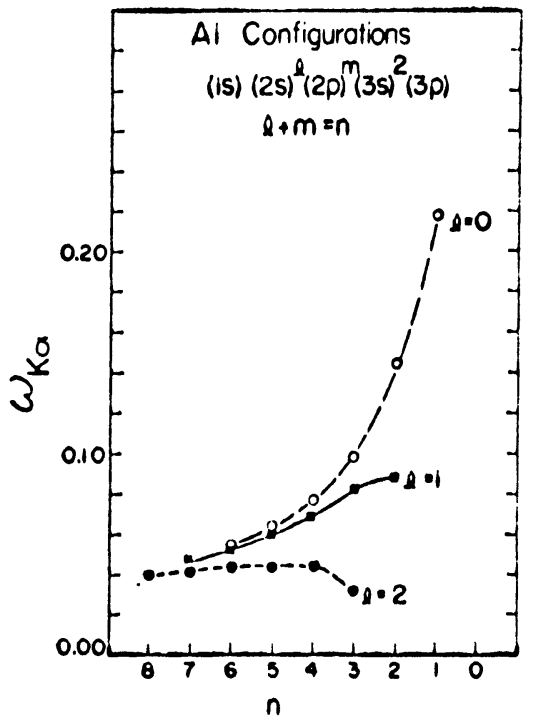

FIG. 24. - Theoretical $\mathbf{K}_{\boldsymbol{\alpha}}$-fluorescence yields for various ionization states of aluminum. The total number of electrons in the L-shell is denoted by $n=l+m$, where $l$ and $m$ are the number of electrons in the $2 s$ and $2 p$ subshells respectively [62].

of X-ray spectra is strictly valid only if transitions from a single initial state occur and if $\omega$ is insensitive to the molecular environment [63].

5.4 The X-ray cross-sections for heavy ion impact exhibit an energy cut-off which has been demonstrated for many combinations of projectile and target [51, 64]. Figure 25 gives an example for this behaviour. Moreover, as the X-ray cross-sections for a particular target may show strong fluctuations due to level matching effects as a function of the atomic number of the impinging projectile $[31,65,66]$, there arises the possibility of exciting characteristic X-rays of an element in a selective manner by bombardment with a suitable projectile at an optimal energy. 


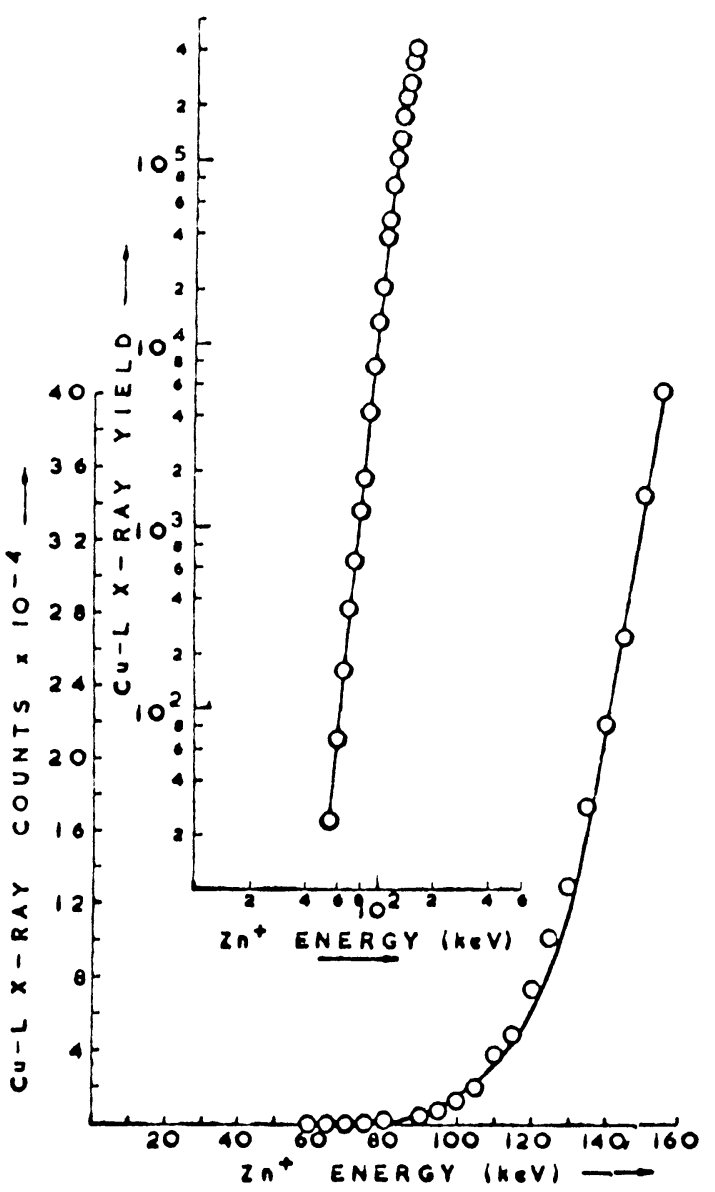

FIG. 25. - CuL-X-ray yield generated from a solid copper target by $\mathrm{Zn}^{+}$projectiles [51].

6. Applications. - During the past few years many papers were published on the application of ioninduced characteristic X-rays in different main fields, namely impurity or trace analysis in solids [3, 67, 69-75], thin film, surface and surface-layer (expecially oxide) studies $[68,76,77]$, depth profile measurements in ionimplanting [79-82]. For a general survey of the range of applications of ion-induced X-rays reference may be made to a paper by J. L. Duggan et al. [67]. Without going into detail, some of the prominent results as to the limitation of sensitivity in trace-analysis should be mentioned. F. Folkmann et al. [75] discuss in some extent the background radiation produced by protons, $\alpha$-particles and oxygen ions in the $\mathrm{MeV} / \mathrm{amu}$ region. It turns out that a main contribution in the $\mathrm{X}$-ray spectra is the bremsstrahlung [83] due to secondary electrons produced in the target by the impinging ions. Figure 26 [75] reveals a higher background of about $50 \%$ in the $\approx 20 \mathrm{keV} \mathrm{X}$-ray photon energy region for $\alpha$ - and oxygen-particles as compared to protons. These authors come to the conclusion that they find no advantage in using high velocities of the projectile for trace-element analysis and for energies of more than $3 \mathrm{MeV} / \mathrm{amu}$ no advantage in using ions heavier than protons. They state that $1-4 \mathrm{MeV}$ protons are the best suited ions for most trace-element pro-

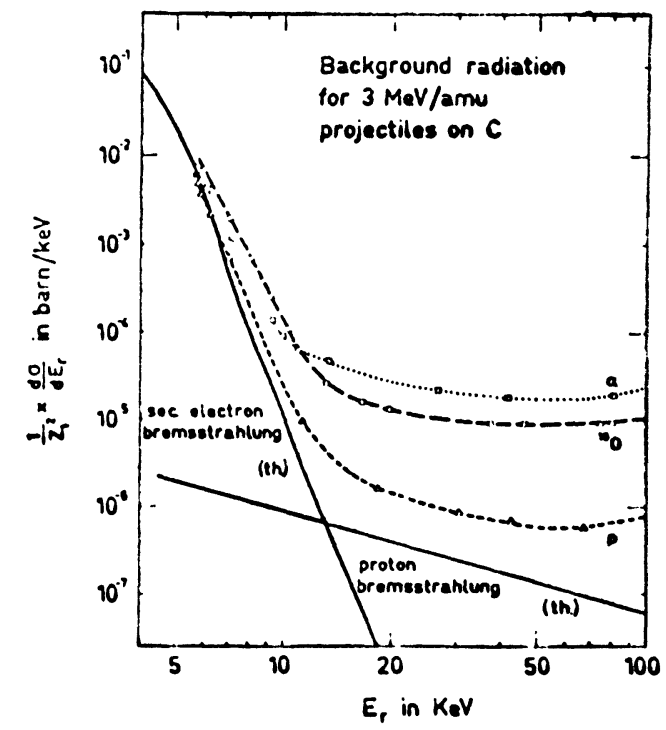

FIG. 26. - Reduced background cross-section from a thin carbon foil [75]. $E_{r}$ : photon energy of the emitted continuous $\mathrm{X}$-rays.

grams. This result is supported by the work of R. P. Chaturvedi et al. [82] who state that in traceelements in infinitely thick targets such as silicon the use of lower energy protons is more advantageous than the use of higher energy oxygen projectiles.

As to the ion-implantation studies T. J. Gray et al. [78] describe sensitivity measurements for As and $\mathrm{Zn}$ implants in Si with 1.4 MeV-protons and $20 \mathrm{MeV}$ $\mathrm{O}^{4+}$-ions. Defining the sensitivity limit as established when the peak channel is within 3 standard deviations of background their data show that this limit is obtained with $2 \times 10^{14}$ implanted $\mathrm{Zn}$ atoms $/ \mathrm{cm}^{2}$ and $2 \times 10^{13}$ implanted As atoms $/ \mathrm{cm}^{2}$ for $1.4 \mathrm{MeV}$-protons. The oxygen data show a marked decrease in sensitivity in spite of an appreciable increase in the $\mathrm{X}$-ray production cross-section for the heavier higher energy ions ; but this increase is offset by a substantial increase in the ion-induced thick target bremsstrahlung, furthermore multiple ionization of $\mathrm{Zn}$ by the oxygen ions causes an observed increase in the $\mathrm{K}_{\alpha}$-X-ray line width by a factor of approximately 2 ; this leads to a reduction of the signal-to-noise ratio by a factor 10 changing from $1.4 \mathrm{MeV}$ protons to $20 \mathrm{MeV}-\mathrm{O}^{4+}$-ions.

7. Conclusion. - In the light of the recent work the promising predictions of the application of ioninduced X-rays to spectrochemical analysis cited in earlier papers must be looked at with some caution. These predictions were substantially due to a preoccupation with the "magnitude of the ion-excitation cross-sections, neglecting the signal-to-noise ratio which ultimately limits the sensitivity of the method. The signal-to-noise ratio depends critically on the chosen projectile-target combination and impact energy [81], thus requiring detailed knowledge for a useful application under the various experimental conditions. 
Comparative studies such as those by J. A. Cooper et al. [72] who compared photon-excitation with proton- and $\alpha$-particle excitation with the result that low energy proton excitation techniques do not exhibit analysis capabilities significantly different from those of photon-excitation methods while high energy $\alpha$-excitation is not competitive.

Much work remains to be done to establish the domains in which the application of ion induced $\mathrm{X}$-rays represents the superior method.

\section{References}

[1] Merzbacher, E. and Lewis, H. W., Encyclopedia of Physics, ed. by Flügge (S.), (Springer Verlag, Berlin), 1958 vol. 34 , p. 166.

[2] Sterk, A. A., Advances in X-ray Analysis, vol. 8, p. 189 (1965).

[3] Johansson, T. B., Akselsson, R. and Johansson, S. A. E., Advances in X-ray Analysis, vol. 15, p. 373 (1972).

[4] Birks, L. S., Seebold, R. E., Batt, A. P. and Grosso, J. S., J. Appl. Phys. 35 (1964) 2578.

[5] Poole, D. M. and Shaw, J. L., Proc. of the 5th Int. Congress on X-Ray Optics and Microanalysis, eds. by Möllenstedt (G.) and Gaukler (K. H.), (Springer Verlag, Berlin) 1969, p. 319.

[6] Harrison, K. G., Tawara, H. and De Heer, F. J., Physica 66 (1973) 16.

[7] Langenberg, A. and VAN EcK, J., Phys. Rev. Lett. 31 (1973) 71.

[8] Scharnagl, T., Diplomarbeit Würzburg 1974.

[9] Saris, F. W. and Onderlinden, D., Physica 49, (1970) 441.

[10] Foster, C., Hoogkamer, T. and Saris, F. W., J. Phys. B 7 (1974) 2563.

[11] Fano, U. and Lichten, W., Phys. Rev. Lett. 14 (1965) 627.

[12] Fink, R. W., Manson, S. T., Palms, J. M. and VenugoPAla RAO, P. (eds), Proc. Int. Conf. Inn. Shell Ioniz. Phen. and Fut. App., USA EC Report No. Conf. 720404 (1973).

[13] Garcia, J. D., Fortner, R. J. and Kavanagh, T. M., Rev. Mod. Phys. 45 (1973) 111

[14] SARIS, F. W., in [12], p. 1269.

[15] SAris, F. W., Atomic Collisions in Solids vol. 1, p. 350, Datz S., Appleton B. R. and Moak C. D. (eds.), (Plenum Press, New York), 1975.

[16] Gray, T. J., Lear, R., Dexter, R. J., Schwettmann, F. N. and WIEMER, K. C., Thin Solid Films 19 (1973) 103.

[17] Chaturvedi, R. P., Duggan, J. L., Gray, T. J., SachtLEBEN, C. C. and LIN, J., Advances in X-Ray Analysis, vol. 17, p. 445, 1974.

[18] Lewis, C. W., Watson, R. L. and Natowitz, J. B., Phys. Rev. A 5, (1972) 1773

[19] Shima, K., Sakisaka, M., Kokado, M., Yamamoto, T. and Makino, I., Japan. J. Appl. Phys. 9 (1970) 1297.

[20] AitKen, D. W., IEEE Trans. Nucl. Sci. NS 15 (1968) 10.

[21] Merzbacher, E., in [12], p. 915.

[22] BRANDT, W., in [12], p. 948.

[23] Saris, F. W., VAN DER WeG, W. F., Tawara, H. and LaUBERT, R., Phys. Rev. Lett. 28 (1972) 717.

[24] Khandei, G. S., ChoI, B. H. and Merzbacher, E., Atomic Data 1 (1969) 103.

[25] Basbas, G., Brandt, W. and Laubert, R., Phys. Rev. A 7 (1973) 983.

[26] Mc Guire, J. H. and Richard, P., Phys. Rev. A 8 (1973) 1374.

[27] Bang, J. and Hansteen, J. M., Kgl. Danske Vid. Selsk. Mat. Fys. Medd. 31 (1959) No. 13.

[28] Brandt, W., Laubert, R. and Sellin, I., Phys. Rev. 151 (1966) 56.

[29] Garcia, J. D., Phys. Rev. A 1 (1970) 280 and 1402.

[30] Garcia, J. D., Phys. Rev. A 4 (1971) 955.

[31] SARIs, F. W., Physica 52 (1971) 290.

[32] Lichten, W. Phys. Rev. 164 (1967) 131.

[33] Barat, M. and Lichten, W., Phys. Rev. A 6 (1972) 211.
[34] Briggs, J. S. and Macek, J., J. Phys. B 5 (1972) 579.

[35] BRIGGS, J. S., Atomic Collisions in Solids, vol. 1, p. 365, Datz, S., Appleton B. R. and Moak C. D. (eds.), (Plenum Press, New York) 1975.

[36] Fortner, R. J., Curry, B. P., Der, R. C., Kavanagh, T. M. and Khan, J. M., Phys. Rev. 185 (1969) 164.

[37] Larkins, F. P., J. Phys. B 5 (1972) 571.

[38] Mott, N. F. and Massey, H. S. W., The Theory of Atomic Collisions (Oxford U. P., London) 1965.

[39] Kessel, Q. C., Bull. Am. Phys. Soc. 14 (1969) 946.

[40] Lin, J., Duggan, J. L. and Carlton, R. F., in [12], p. 998.

[41] Bissinger, G. A., Joyce, J. M., Ludwig, E. J., MCEver, W. S. and Shafroth, S. M., Phys. Rev. A 1 (1970) 841.

[42] Needham, P. B. and Sartwell, B. D., Phys. Rev. A 2 (1970) 1686.

[43] Needham, P. B. and Sartwell, B. D., Advances in X-ray Analysis, vol. 14, eds. by Barrett (C. S.), Newkirk (J. B.) and Rud (C. O.) (Plenum Press, New York) (1971), 184

[44] Knudson, A. R., Nagel, D. J., Burkhalter, P. G. and Dunning, K. L., Phys. Rev. Lett. 26 (1971) 1149.

[45] LaRkins, F. P., in [12], p. 1543.

[46] RuDD, M. E., in [12], p. 1485.

[47] Aberg, T., in [12], p. 1509.

[48] Blokhin, M. A., Sachenko, V. P. and Schweizer, I. G., in [12], p. 1696

[49] Nigam, A. N. and Mathur, R. B., in [12], p. 1698.

[50] Kamada, H., Tamura, T. and Terasawa, M., Sixth Int. Conf. on X-Ray Optics and Microanalysis, Shinoda (G.), Kohra (K.) and Ichinokawa (T.) (eds.) (University of Tokyo Press) 1972, p. 541.

[51] Cairns, J. A., Marwick, A. D. and Mitchell, I. V., Thin Solid Films 19 (1973) 91.

[52] Saris, F. W., Van der Weg, W. F., Tawara, H. and LAUBert, R., Phys. Rev. Lett. 28 (1972) 717.

[53] Saris, F. W., Mitchell, I. V., SANTry, D. C., Davies, J. A and LAUBERT, R., in [12], p. 1255.

[54] Toburen, L.H., Phys. Rev. A 5 (1972) 2482

[55] Larkins, F. P., J. Phys. B 4 (1971) L 29.

[56] Fortner, R. J., Der, R. C., Kawanagh, T. M. and Garcia, J. D., J. Phys. B 5 (1973) L 73.

[57] Toburen, L. H. and Larkins, F. P., Phys. Rev. A 6, (1972) 2035.

[58] Bhalla, C. P., Phys. Lett. 45A (1973) 19.

[59] Bhalla, C. P., Atomic Collisions in Solids, vol. 1, p. 407, Datz (S.), Appleton (B. R.) and Moak (C. D.), (eds.) (Plenum Press New York) 1975.

[60] Bhalla, C. P., Folland, N. O., Heain, M. A., Phys. Rev. A 8 (1973) 649.

[61] Saris, F. W. and Bhalla, C. P., J. Phys. B 7 (1974) L 115.

[62] Bhalla, C. P. and Richard, P., Phys. Lett. 45A (1973) 53.

[63] Harison, K. G., Tawara, H., De Heer, F. J. and SARIS, F. W., in [12], p. 414.

[64] Cairns, J. A., Holloway, D. F. and Nelson, R. S., Advances in X-Ray Analysis, vol. 14, eds. by Barrett (C. S.), Newkirk (J. B.) and Rund (C. O.), (Plenum Press, New York), 1971, p. 173.

[65] Kavanagh, T. M., Cunningham, M. E., Der, R. C., FORTNER, R. J., KHAN, J. M., ZAHARIS, E. J. and GARCIA, J. D., Phys. Rev. Lett. 25 (1970), 1473. 
[66] Brandt, W. and Laubert, R., Phys. Rev. Lett. 24 (1970) 1037.

[67] Duggan, J. L., Beck, W. L., Albrecht, L., Munz, L. and Spaulding, J. D., Advances in X-ray Analysis, vol. 15, (1972) 407.

[68] Musket, R. G. and Bauer, W., J. Appl. Phys. 43 (1972) 4786.

[69] Umbarger, C. J., Bearse, R. C., Close, D. A. and Malanity, J. J., Advances in X-ray Analysis, vol. 16, p. 102, (1973).

[70] Campbell, J. L., Herman, A. W., Mc Nelles, L. A., Orr, B. H. and Willoughby, R. A., Advances in $X$-ray Analysis, vol. 17, p. 457 (1974).

[71] Shabason, L., Cohen, B. L., Wedberg, G. H. and Chan, K. C., J. Appl. Phys. 44 (1973) 4749.

[72] CoOper, J. A., Nucl instrum. Meth. 106 (1973) 525.

[73] Herman, A. W., Mc Nelles, L. A. and Campbell, J. L., Nucl. Instrum. Meth. 109 (1973) 429.

[74] Valković, V., Liebert, R. B., Zabel, T., Larson, H. T., Miljanić, D., Wheeler, R. M. and Phillips, G. C., Nucl. Instrum. Meth. 114 (1974) 574.
[75] Folkmann, F., Borggreeis J. and KjeldgaArd, A., Nucl. Instrum. Meth. 119, (1974) 117.

[76] Feldmann, L. C., Poate, J. M., Ermanis, F. and Schwartz, B., Thin Solid Films 19 (1973) 81.

[77] Musket, R. G. and Bauer, W., Thin Solid Films 19 (1973) 69.

[78] Gray, T. J., Lear, R., Dexter, R. J., Schwettmann, F. N. and Wiemer, K. C., Thin Solid Films 19 (1973) 103.

[79] Nelson, R. S., Cairns, J. A. and Blamires, N., Radiat. Eff. 6 (1970) 131.

[80] Cairns, J. A., Holloway, D. F. and Nelson, R. S., Europ. Conf. on Ion Implantation, Reading (England) Peter Peregrinus (1970), 203.

[81] Chemin, J. F., Mitchell, I. V. and Saris, F. W., J. Appl. Phys. 45 (1974) 532, 537.

[82] Chaturvedi, R. P., Duggan, J. L., Gray, T. J. and SaChtleben, C. C. and Lin, J., Advances in X-ray Analysis, vol. 17, (1974) 445.

[83] Folkmann, F., GaArde, C., Huus, T. and Kemp, K., Nucl. Instrum. Meth. 116 (1974) 487. 